Bicocca-FT-03-5

TTP03-07

UND-HEP-02-BIG08

hep-ph/0302262

\title{
Imprecated, yet Impeccable: On the Theoretical Evaluation of $\Gamma\left(B \rightarrow X_{c} \ell \nu\right)$
}

\author{
Submitted to the Proceedings of the CKM Workshop \\ D. Benson ${ }^{a, b}$, I.I. Bigi ${ }^{a, b}$, Th. Mannel ${ }^{b}$ and N. Uraltsev ${ }^{c *}$ \\ ${ }^{a}$ Department of Physics, University of Notre Dame du Lac \\ Notre Dame, IN 46556, USA ${ }^{1}$ \\ ${ }^{b}$ Institut für Theoretische Teilchenphysik, Universität Karlsruhe \\ D-76128 Karlsruhe, Germany \\ ${ }^{c}$ INFN, Sezione di Milano, Milan, Italy
}

\begin{abstract}
We present a detailed evaluation of the total semileptonic $B$ meson width in terms of $\left|V_{c b}\right|$ and heavy quark parameters (quark masses and the expectation values of local heavy quark operators). Special attention is given to perturbative corrections which can precisely be calculated in a scheme with a hard Wilsonian cutoff at a scale around $1 \mathrm{GeV}$ appropriate for the OPE, and to the potential impact of higher-order power corrections. We point out that the latter require control over possible contributions from four-quark operators containing charm quark fields. Analytical expressions are given which allow evaluating the width with various choices of parameters; ready-to-use expressions showing the dependence on the heavy quark parameters are presented as well. We illustrate these results by commenting on how these parameters can be extracted and what accuracy is likely to be achievable in the near future.
\end{abstract}

\footnotetext{
${ }^{1}$ Permanent address

* On leave of absence from Department of Physics, University of Notre Dame, Notre Dame, IN 46556, USA and St. Petersburg Nuclear Physics Institute, Gatchina, St. Petersburg 188300, Russia
} 


\section{Contents}

1 Executive Summary 2

$2 \Gamma\left(B \rightarrow X_{c} \ell \nu\right) ;$ Master Formulae $\quad 4$

3 Heavy Quark Parameters $\quad 7$

3.1 Heavy quark masses . . . . . . . . . . . . . . . . . . . . . 7

3.2 Operators of dimension 5 and $6 \ldots \ldots \ldots \ldots \ldots$

3.3 Operators with charm quarks $\ldots \ldots \ldots \ldots \ldots$

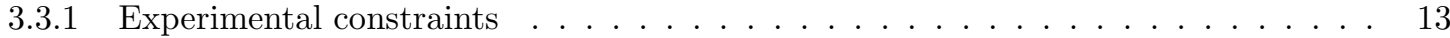

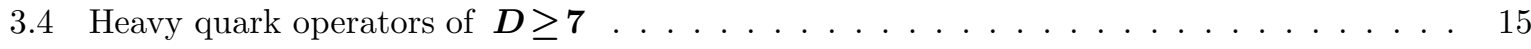

4 Perturbative Contributions 19

$4.0 .1 \quad$ BLM summation . . . . . . . . . . . . . . . . . . . . . . . . . . 19

4.0 .2 Non-BLM contributions . . . . . . . . . . . . . . . . . . . . . 20

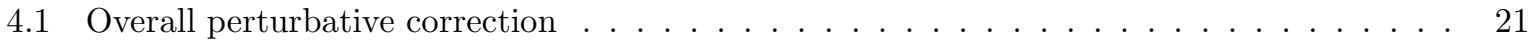

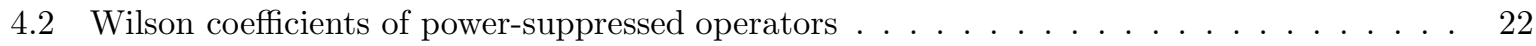

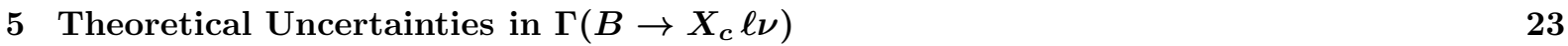

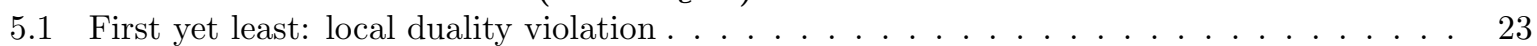

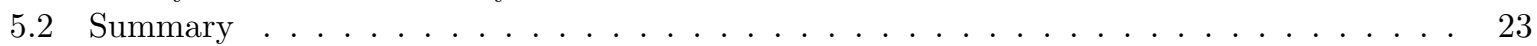

6 Determining the Heavy Quark Parameters 24

6.1 Numerical examples . . . . . . . . . . . . . . . . . . . . . . . 26

$\begin{array}{lll}7 & \text { Summary and Outlook } & 26\end{array}$

$\begin{array}{ll}\text { Appendices } & \mathbf{2 8}\end{array}$

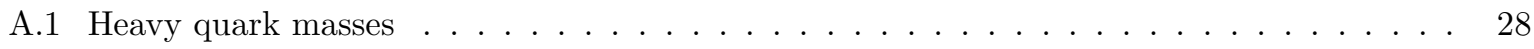

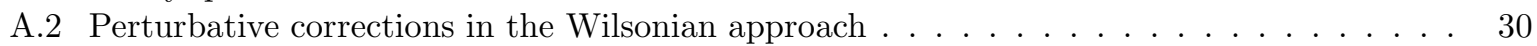

A.3 BLM summation with Wilsonian cutoff . . . . . . . . . . . . . . . . . 31

Extracting the value of $\left|V_{c b}\right|$ from the observed semileptonic width of $B$ mesons constitutes a method of impeccable theoretical pedigree [1], although it often has been imprecated in the past. The theoretical part of the analysis proceeds in two steps:

1. Through a heavy quark expansion (HQE) one expresses $\Gamma\left(B \rightarrow l \nu X_{c}\right)$ as $\left|V_{c b}\right|^{2}$ times a function of the heavy quark (HQ) parameters like quark masses and hadronic expectation values, and the perturbative contributions.

2. One determines the values of these HQ parameters from observables other than the total semileptonic width.

The first step has now reached a mature stage after having been subjected to considerable scrutiny for several years. The primary motivation for this note is to present an updated description of this first task. We will give analytical expressions as much as reasonably possible, state explicitly the proper field theoretical definitions of the HQ parameters and address the potential sources for theoretical uncertainties that arise in such an expansion. We aim at giving an 'open source code' which enables the dedicated reader to check the validity of the theoretical results and insert personally preferred values for the $\mathrm{HQ}$ parameters and incorporate additional constraints.

To extract a value $\left|V_{c b}\right|$ from the observed width one has, of course to complete step two as well. There exists considerable information and constraints on these HQ parameters. We will sketch how they 
can be determined. However, more work is still needed. We will present numbers in this context; yet they are to be understood as illustrative rather than final.

There is already highly nontrivial evidence that the systematics are indeed under control. We anticipate that in the end one will be able to reduce the overall theoretical uncertainty to the one percent level. This benchmark will guide us in calculating $\Gamma_{\mathrm{sl}}(B)$.

The remainder of this note will be organized as follows: in Sect. 1 we provide a summary of the expressions that one can use to obtain the value of $\left|V_{c b}\right|$ from the total semileptonic $B$ width. In Sect. 2 we present the theoretical expressions used to derive the total semileptonic $B$ width and discuss all ingredients in the corresponding subsections. We include the effects of four-quark operators with charm field previously ignored, and analyze the potential effect of higher order power corrections. Then we discuss in more detail the definitions and basic properties of the heavy quark operators, and address the perturbative corrections in Sect. 4. Section 5 summarizes the theoretical uncertainties in evaluating $\Gamma_{\mathrm{sl}}$. In Sect. 6 we illustrate how one can independently determine the values of the required heavy quark parameters from experiment. Technical details are relegated to Appendices, where we give explicit expressions for the normalization point dependence of the heavy quark parameters, provide formulae for computing perturbative corrections implementing Wilsonian OPE, and briefly describe the BLM summation to arbitrary order within this framework.

\section{Executive Summary}

We base our numerical analysis on the following expression for the semileptonic $B$ width [2] through order $1 / m_{Q}^{3}$ :

$$
\begin{aligned}
\Gamma_{\mathrm{sl}}(b \rightarrow c) & =\frac{G_{F}^{2} m_{b}^{5}(\mu)}{192 \pi^{3}}\left|V_{c b}\right|^{2}\left(1+A_{\mathrm{ew}}\right) A^{\mathrm{pert}}(r, \mu)\left[z_{0}(r)\left(1-\frac{\left.\mu_{\pi}^{2}(\mu)-\mu_{G}^{2}(\mu)+\frac{\rho_{D}^{3}(\mu)+\rho_{L S}^{3}(\mu)}{m_{b}(\mu)}\right)}{2 m_{b}^{2}(\mu)}\right)\right. \\
& \left.-2(1-r)^{4} \frac{\mu_{G}^{2}(\mu)-\frac{\rho_{D}^{3}(\mu)+\rho_{L S}^{3}(\mu)}{m_{b}(\mu)}}{m_{b}^{2}(\mu)}+d(r) \frac{\rho_{D}^{3}(\mu)}{m_{b}^{3}(\mu)}+\ldots\right]
\end{aligned}
$$

where $z_{0}(r)$ is the tree-level phase space factor and $r=m_{c}^{2}(\mu) / m_{b}^{2}(\mu)$ :

$$
z_{0}(r)=1-8 r+8 r^{3}-r^{4}-12 r^{2} \ln r
$$

while the expression for $d(r)$ follows from Ref. [3]:

$$
d(r)=8 \ln r+\frac{34}{3}-\frac{32}{3} r-8 r^{2}+\frac{32}{3} r^{3}-\frac{10}{3} r^{4} \simeq-18.3 z_{0} \text { at } \sqrt{r}=0.25 .
$$

The electroweak correction, $A_{\mathrm{ew}}$ corresponding to the ultraviolet renormalization of the Fermi interaction is well-known [4]:

$$
1+A_{\mathrm{ew}} \simeq\left(1+\frac{\alpha}{\pi} \ln \frac{M_{Z}}{m_{b}}\right)^{2} \simeq 1.014
$$

An auxiliary scale $\mu$ is introduced to demark the border between long- and short-distance dynamics in the OPE. Unless stated otherwise, we adopt $\mu \simeq 1 \mathrm{GeV}$. 
The quantity $A^{\text {pert }}$ accounts for the perturbative contributions; it has been calculated to all orders in BLM corrections and to second order in non-BLM corrections as discussed in detail in Sect. 2. For $\sqrt{r}=0.25$ and $\mu=1 \mathrm{GeV}$ we find that $A^{\text {pert }} \simeq 0.908$.

The quantities $\mu_{\pi}^{2}, \mu_{G}^{2}, \rho_{D}^{3}$ and $\rho_{L S}^{3}$ denote the expectation values of the kinetic, chromomagnetic, Darwin and spin-orbit operators, respectively. Only $\rho_{D}^{3}$ has a noticeable impact at $\mathcal{O}\left(1 / m_{b}^{3}\right)$. In that order in $1 / m_{b}$ there arise also contributions corresponding to four-quark operators of the generic form $(\bar{b} \Gamma c)(\bar{c} \Gamma b)$. Their nonperturbative $B$ expectation value, while small, will not vanish. For now we include such a contribution in our estimate of the theoretical uncertainty, given below.

Using the numerical values $m_{b}(1 \mathrm{GeV})=4.6 \mathrm{GeV}, \mu_{\pi}^{2}(1 \mathrm{GeV})=0.4 \mathrm{GeV}^{2}, \mu_{G}^{2}(1 \mathrm{GeV})=$ $0.35 \mathrm{GeV}^{2}, \rho_{D}^{3}(1 \mathrm{GeV})=0.2 \mathrm{GeV}^{3}$ and $\rho_{L S}^{3}(1 \mathrm{GeV})=-0.15 \mathrm{GeV}^{3}$, for $\sqrt{r}=0.25$ and $\alpha_{s}\left(m_{b}\right)=0.22$ we find the following expression for $\left|V_{c b}\right|:^{1}$

$$
\frac{\left|V_{c b}\right|}{0.0417} \simeq\left(\frac{\mathrm{Br}_{\mathrm{sl}}(B)}{0.105}\right)^{\frac{1}{2}}\left(\frac{1.55 \mathrm{ps}}{\tau_{B}}\right)^{\frac{1}{2}}\left(1-4.8\left[\operatorname{Br}\left(B \rightarrow X_{u} \ell \nu\right)-0.0018\right]\right)\left(1+\delta_{\mathrm{th}}\right),
$$

with the following dependence of $\left|V_{c b}\right|$ on the various heavy quark parameters:

$$
\begin{aligned}
\frac{\left|V_{c b}\right|}{0.0417}=\left(1+\delta_{\text {th }}\right)[1+0.30 & \left.\left(\alpha_{s}\left(m_{b}\right)-0.22\right)\right] \times \\
{[1} & -0.66\left(m_{b}(1 \mathrm{GeV})-4.6 \mathrm{GeV}\right)+0.39\left(m_{c}(1 \mathrm{GeV})-1.15 \mathrm{GeV}\right) \\
+ & 0.013\left(\mu_{\pi}^{2}-0.4 \mathrm{GeV}^{2}\right)+0.09\left(\rho_{D}^{3}-0.2 \mathrm{GeV}^{3}\right) \\
+ & \left.0.05\left(\mu_{G}^{2}-0.35 \mathrm{GeV}^{2}\right)-0.01\left(\rho_{L S}^{3}+0.15 \mathrm{GeV}^{3}\right)\right] .
\end{aligned}
$$

Beyond the uncertainties in the numerical values of these parameters there is the potential error in $\left|V_{c b}\right|$ due to the limited accuracy of the theoretical expression for the semileptonic width we relied upon; this is denoted by $\delta_{\text {th }}$, for which we estimate

$$
\delta_{\text {th }}= \pm 0.005_{\text {pert }} \pm 0.012_{\mathrm{hWc}} \pm 0.004_{\mathrm{hpc}} \pm 0.007_{\mathrm{IC}} .
$$

The terms here represent the remaining uncertainty in the Wilson coefficient of the leading operator $\bar{b} b$ (perturbative correction), as yet uncalculated perturbative corrections to the Wilson coefficients of chromomagnetic and Darwin operators, higher-order power corrections including violation of local duality in $\Gamma_{\mathrm{sl}}(b \rightarrow c)$, and possible nonperturbative effects in the operators with charm fields, respectively.

While the numbers of Eq. (7) are small, we consider them rather on the conservative side. Yet they should be viewed as what they are, namely estimates based on best efforts rather than mathematical theorems.

\footnotetext{
${ }^{1}$ The number slightly differs from Ref. [5] since we adopt the Wilsonian renormalization convention for Darwin term as well; our $\rho_{D}^{3}(\mu)$ should be distinguished from $\tilde{\rho}_{D}^{3}$ of Refs. [5, 6].
} 


\section{$2 \Gamma\left(B \rightarrow X_{c} \ell \nu\right) ;$ Master Formulae}

The operator product expansion (OPE) yields inclusive heavy quark decay rates as an asymptotic series in inverse powers of the heavy quark mass. To be more precise, there are different mass scales in the problem; the most important one is the energy release given by $E_{r}=m_{b}-m_{c}$ for $b \rightarrow c[1]$. Hence, the width in the OPE is an expansion in inverse powers of $E_{r}{ }^{2}$ It is useful to note that for actual quark masses the dependence on $m_{b}$ and $m_{c}$ is well approximated by $\Gamma_{\mathrm{sl}}(B) \propto m_{b}^{2}\left(m_{b}-m_{c}\right)^{3}$.

Through order $1 / m_{Q}^{3}$ one has the general expression

$$
\begin{aligned}
\Gamma_{\mathrm{sl}}(b \rightarrow c) & =\frac{G_{F}^{2} m_{b}^{5}(\mu)}{192 \pi^{3}}\left|V_{c b}\right|^{2}\left(1+A_{\mathrm{ew}}\right)\left[z_{0}(r)\left[1+A_{3}^{\mathrm{pert}}(r ; \mu)\right]\left(1-\frac{\left.\mu_{\pi}^{2}(\mu)-\mu_{G}^{2}(\mu)+\frac{\rho_{D}^{3}(\mu)+\rho_{L S}^{3}(\mu)}{m_{b}(\mu)}\right)}{2 m_{b}^{2}(\mu)}\right)\right. \\
& -\left(1+A_{5}^{\text {pert }}(r ; \mu)\right) 2(1-r)^{4} \frac{\mu_{G}^{2}(\mu)-\frac{\rho_{D}^{3}(\mu)+\rho_{L S}^{3}(\mu)}{m_{b}(\mu)}}{m_{b}^{2}(\mu)}+\left(1+A_{D}^{\text {pert }}\right) d(r) \frac{\rho_{D}^{3}(\mu)}{m_{b}^{3}(\mu)} \\
& +32 \pi^{2}\left(1+A_{6 c}^{\text {pert }}(r)\right)(1-\sqrt{r})^{2} \frac{H_{c}}{m_{b}^{3}(\mu)}+32 \pi^{2} \tilde{A}_{6 c}^{\text {pert }}(r)(1-\sqrt{r})^{2} \frac{\tilde{H}_{c}}{m_{b}^{3}(\mu)} \\
& \left.+32 \pi^{2} A_{6 q}^{\text {pert }}(r) \frac{F_{q}}{m_{b}^{3}(\mu)}+\mathcal{O}\left(\frac{1}{m_{b}^{4}}\right)\right],
\end{aligned}
$$

The phase space factor $z_{0}(r)$ has been defined in Eq. (2) and the tree coefficient for the Darwin term $d(r)$ in Eq. (3). A number of clarifications are to be made about the above master expression.

Following Wilson's prescription for the OPE an auxiliary scale $\mu$ has been introduced to separate the effects from long- and short-distance dynamics. Since observables do not depend on it, quark masses, radiative corrections and hadronic expectation values $\left\langle B\left|O_{i}\right| B\right\rangle$ combine to yield a compensating $\mu$ dependence as indicated in Eq. (8). The natural and most efficient choice is $\mu \simeq 1 \mathrm{GeV}$, which is particularly relevant for the quark masses [8].

It should be kept in mind that the $B$ state over which the expectation values are taken is the real $B$ meson, not the meson in the heavy quark limit $m_{b} \rightarrow \infty$. In this convention the total width to order $1 / m_{b}^{3}$ is corrected by a single Darwin expectation value (modulo the effect of four-quark operators to be addressed below). No non-local correlators enter, and the spin-orbital expectation value $\rho_{L S}^{3}$ enters only as a $1 / m_{b}$ piece of the total Lorentz-scalar chromomagnetic expectation value [9]. ${ }^{3}$ Likewise, the kinetic

\footnotetext{
${ }^{2}$ While the OPE in Eqs. (1), (8) at first sight look like expansion in powers of $1 / m_{b}$, this is not really the case. In the SV limit $m_{b}-m_{c} \ll m_{b}$ the expansion parameter is obviously $1 /\left(m_{b}-m_{c}\right)$; at $m_{b} \rightarrow m_{c}$ the expansion becomes meaningless no matter how large $m_{b}$ is. Constructing the OPE directly for the widths shows that the expansion parameter in general is $1 /\left(m_{b}-m_{c}\right)$ [7], which comes about in Eqs.(1), (8) due to the interplay of $1 / m_{b}$ and $r=m_{c}^{2} / m_{b}^{2}$. On the other hand expectation values like $\langle B|\bar{b} b| B\rangle$ are expanded in powers of $1 / m_{b}$.

${ }^{3}$ The inclusive moments of various decay distributions do exhibit explicit dependence on $\rho_{L S}^{3}$; it is however too weak to matter in practice.
} 
expectation value enters solely through the $1 / m_{b}$ expansion of the leading operator $\bar{b} b$ $[10,2,9]$.

All Wilson coefficients are given by short-distance dynamics and in practice are evaluated in perturbation theory. In the convention adopted in Eq. (8) the tree-level coefficients $z_{0}, d$ etc. are modified by perturbative corrections $A_{\ldots}^{\text {pert }}$ starting with at least one power of $\alpha_{s}$.

The leading nonperturbative corrections arise in order $1 / m_{Q}^{2}$ and are controlled by the expectation values $\mu_{\pi}^{2}(\mu)$ and $\mu_{G}^{2}(\mu)$ of the kinetic and chromomagnetic dimension-five operators $\left(D_{\nu}\right.$ denotes the covariant derivative), respectively

$$
\mu_{\pi}^{2}(\mu) \equiv \frac{1}{2 M_{B}}\left\langle B\left|\bar{b}(i \vec{D})^{2} b\right| B\right\rangle_{\mu}, \quad \mu_{G}^{2}(\mu) \equiv \frac{1}{2 M_{B}}\left\langle B\left|\bar{b} \frac{i}{2} \sigma_{j k} G^{j k} b\right| B\right\rangle_{\mu}
$$

(Since we have explicitly introduced the effect of the $L S$ operator, $\mu_{G}^{2}$ above should be understood as the average of only the chromomagnetic field, $\langle\vec{\sigma} \vec{B}\rangle$, although it includes $1 / m_{b}$ finite-mass effects.) The Darwin and spin-orbital $L S$ terms $\rho_{D}^{3}(\mu)$ and $\rho_{L S}^{3}(\mu)$ emerge from dimension-six operators:

$$
\rho_{D}^{3}(\mu) \equiv \frac{1}{2 M_{B}}\left\langle B\left|\vec{b}\left(-\frac{1}{2} \vec{D} \cdot \vec{E}\right) b\right| B\right\rangle_{\mu}, \quad \rho_{L S}^{3}(\mu) \equiv \frac{1}{2 M_{B}}\langle B|\vec{b}(\vec{\sigma} \cdot \vec{E} \times i \vec{D}) b| B\rangle_{\mu}
$$

The Wilson coefficients for the operators describing nonperturbative effects are completely known only at tree level. Since they represent power-suppressed effects showing up at the one percent level, this is a reasonable approximation. However, it is desirable to improve it in the future computing $\mathcal{O}\left(\alpha_{s}\right)$ corrections to them.

The last term in Eq. (8) proportional to $F_{q}$ denotes the effect of generic $S U(3)$-singlet four-quark operators, other than Darwin operator, of the form $\bar{b} \Gamma \bar{q} \Gamma q$ with the sum over $q=u, d, s$, and $\Gamma$ including both color and Lorentz matrices (to the leading order in $1 / m_{b}$ only $\gamma_{0} \times \gamma^{0}$ or $\gamma_{i} \gamma_{5} \times \gamma^{i} \gamma_{5}$ structures survive, but one does not need to rely on this). Their Wilson coefficients are order $\mathcal{O}\left(\alpha_{s}\right)$, and we neglect these contributions.

On the other hand, we have explicitly included in the master equation a possible effect of the (tree level) expectation values of the four-quark operators with the charm field. It is routinely skipped assuming that it vanishes due to the sizeable charm mass. While it represents a reasonable approximation, it can be valid only up to a certain accuracy. Since the Wilson coefficient of one linear combination of four (in the heavy- $b$ limit) such operators emerges at tree level and is strongly enhanced by the effectively two-body phase space, this effect might not be totally negligible. To complement its purely theoretical estimates or upper bounds, we find it advantageous to explicitly introduce this effect and study its possible manifestations in experiment:

$$
H_{c}=\frac{1}{2 M_{B}}\left\langle B\left|\bar{b} \gamma_{\nu}\left(1-\gamma_{5}\right) c \bar{c} \gamma^{\rho}\left(1-\gamma_{5}\right) b\right| B\right\rangle_{\mu}\left(-\delta_{\rho}^{\nu}+v^{\nu} v_{\rho}\right), \quad v_{\nu}=\frac{P_{\nu}^{B}}{M_{B}}
$$

This way to refer to such nonperturbative effects literally applies when normalization point $\mu$ is taken above $m_{c}$. Having the normalization scale $\mu$ below $m_{c}$ amounts to the $c$ 
quark field being integrated out; then there are no dynamical $c$ quark fields. Nevertheless, the physical effects lumped into $H_{c}$ still do not have to vanish exactly; they are rather partially reshuffled into higher-dimension b-quark operators with only light fields.

The second charm-related $D=3$ parameter $\tilde{H}_{c}$ in Eq. (8) represents the cumulative effect of the remaining combinations of the four-fermion operators with charm quarks. It is expected to play an insignificant role, but will be briefly addressed as well.

It turns out that the consistent $1 / m_{b}$ expansion to higher orders mandates introducing such four-quark charmed operators. They can be excluded only at the price of spoiling the expansion: instead of $1 / m_{b}$ or $1 / E_{r}$ some of the higher-dimensional operators would get powers of $m_{c}$ in the denominator to compensate for the increasing dimension. To state it differently: the Wilson coefficients of the higher-dimensional operator can contain an enhancement by positive powers of $1 / r$. The aggregate effect of such terms can be viewed as the definition of $H_{c}$ at low normalization scale. Moreover, a significant fraction of the numerically enhanced Darwin coefficient function $d(r)$ represents actually the midvirtuality (semi-hard, or hybrid) contribution to the leading charm operator. A detailed discussion of these operators and their relation to the notion of "Intrinsic Charm" will be given in a separate publication [11]. We shall briefly recapitulate the main points of that analysis below in Sect. 3.3.

The Wilsonian separation of "soft" and "hard" effects can be done in different ways. In particular, this affects the precise values of the heavy quark masses at a given $\mu$. We consistently use the scheme based on the Small Velocity, or Shifman-Voloshin (SV) sum rules which introduce the normalization scale $\mu$ via the cutoff over the excitation energy of the hadronic states $[8,12]$. Other possibilities to define heavy quark masses discussed in the literature are considered in the review [13]. ${ }^{4}$ The advantages of this choice are transparent. First, they allow to define the heavy quark masses as well as the expectation values of the higher-dimensional operators on a parallel footing. Secondly, being expressed via (in principle) measurable quantities, they have a definite value in QCD at any given normalization point. The question of their precise extraction from experiment then becomes meaningful (see, e.g. Ref. [14]), without invoking doubtful procedures. Thirdly, such a renormalization scheme respects exact inequalities established in the heavy quark limit (for a recent review, see Ref. [7]), which turn out very constraining in practice and allow to go far beyond naive dimensional estimates even for higher-dimensional operators. Finally, it has been demonstrated in numerous applications that using such a scheme typically yields a much improved convergence of the perturbative expansion, see, e.g. a recent experimental implementation in Ref. [6].

Below we discuss the salient features in more detail.

\footnotetext{
${ }^{4}$ Their assessment in Ref. [13] is not always justified in our opinion.
} 


\section{$3 \quad$ Heavy Quark Parameters}

\subsection{Heavy quark masses}

With the width depending on the fifth power of the heavy quark mass any uncertainty associated with it - conceptual or numerical - has a grave impact on the accuracy with which $\left|V_{c b}\right|$ can be extracted. Therefore great care has to be applied to properly defining it and consistently calculating the radiative corrections.

The pole mass is defined in analogy to the electron case in QED as the position of the pole in the quark Green function. This pole mass is gauge invariant and perturbatively infrared finite. Employing it is often convenient for purely perturbative calculations. Yet in full QCD due to confinement there is no such pole - the pole mass is thus not 'infrared stable' due to nonperturbative dynamics. Moreover, it has an intrinsic and thus irreducible theoretical uncertainty $\sim \Lambda_{\mathrm{QCD}}$ already in perturbation theory; this makes it inappropriate when one wants to include nonperturbative contributions, which are power suppressed [15]:

$$
\Gamma_{\mathrm{sl}}(B) \propto m_{b}^{5} \simeq\left(m_{b}^{\text {pole }}\right)^{5}\left(1+5 \mathcal{O}\left(\Lambda_{\mathrm{QCD}}\right) / m_{b}\right) ;
$$

i.e., the intrinsic uncertainty is at least parametrically larger than the leading nonperturbative contributions that arise in order $1 / m_{b}^{2}$. The meaning of this observation can be easily understood. A long-distance pole mass in perturbation theory is a counterpart of the heavy flavor hadron mass of full QCD, since it includes effects of gluons with arbitrarily small momenta. In this respect the analogue of the quark mass in QCD where nonperturbative effects exit, is a short-distance mass $m_{Q}(\mu)$ of perturbation theory, since such a mass excludes soft gluons interacting strongly. The central OPE result that there are no contributions $\sim \mathcal{O}\left(1 / m_{b}\right)$ contributions to fully inclusive widths $[10,16]-$ in other words, the hadron ( $B$ meson) mass is irrelevant for the width which depends only on $m_{b}$, but not on $M_{B}-m_{b}$ - translates then into the statement that short-distance masses have to be used when aiming at the power-like accuracy.

To a limited extent, the pole mass can still be used to approximate the width: the order-to-order shift in the apparent position of the pole quark mass in perturbation theory is offset by a significant change in the overall perturbative correction factor. Yet such a procedure may not allow to properly account already for the actual leading nonperturbative corrections. This is illustrated by the following simple observation. Starting with the short-distance mass, one has for the leading (parton) contribution

$$
\Gamma_{\mathrm{sl}}(B) \propto A^{\mathrm{pert}}(\mu) m_{b}^{5}(\mu)
$$

with neither factor having uncontrollable infrared pieces. The same perturbative expression for the pole masses then must be of the form

$$
\Gamma_{\mathrm{sl}}(B) \propto A^{\text {pert }}(\mu)\left(1-5 \frac{\tilde{\Lambda}}{m_{b}}\right)\left(m_{b}^{\text {pole }}\right)^{5} \simeq A^{\text {pert }}(\mu) m_{b}^{5}(\mu)\left[1-15\left(\frac{\tilde{\Lambda}}{m_{b}}\right)^{2}\right],
$$

where $\tilde{\Lambda} / m_{b}$ expresses the uncontrollable infrared contributions in the ratio of the pole to short-distance mass. The contribution $\sim \mathcal{O}\left(1 / m_{b}\right)$ has indeed disappeared, yet the 
expression is still deficient: while there are contributions $\sim \mathcal{O}\left(1 / m_{b}^{2}\right)$, they have to be of the form $1-\frac{1}{2} \frac{\mu_{\pi}^{2}}{m_{b}^{2}}$ regardless of the particular interactions. ${ }^{5}$ Hence the last term in Eq. (14) coming with the coefficient 15 has no counterpart in actual QCD and is rather an artefact of using the pole masses.

An example of a short-distance $b$ mass is the $\overline{\mathrm{MS}}$ mass $\bar{m}_{b}\left(m_{b}\right)$. The normalization scale $m_{b}$, however is unnaturally high for the problem; therefore it introduces significant higher-order corrections not related to running of $\alpha_{s}$ per se, which are under poor control and thus limit the accuracy [8].

A better choice when treating $B$ decays is taking the Wilsonian factorization scale $\mu$ around $1 \mathrm{GeV}$. An example of such a mass is the one defined through the SV sum rules, the prescription valid to arbitrary perturbative order. It has the meaning of the mass entering the kinetic energy of the heavy quark and is often called kinetic mass $m_{b}^{\mathrm{kin}}(\mu)$. At every given $\mu$ it represents an observable physical quantity and, with an appropriate choice of $\mu$ its extraction from experiment provides good stability with respect to radiative corrections. Its definition is discussed in Sect. A.1. Here we mention that its value has been extracted from the threshold domain of the $e^{+} e^{-}$annihilation into $\bar{b} b$,

$$
m_{b}^{\text {kin }}(1 \mathrm{GeV}) \simeq(4.57 \pm 0.06) \mathrm{GeV}
$$

From now on we omit the superscript "kin" using this mass as the default definition.

For the charm quark, the separation scale taken around $1 \mathrm{GeV}$ is similar in magnitude to $m_{c}$ itself. The kinetic mass $m_{c}(\mu)$ can still be used, with the realization, however, that this definition starts loosing direct physical meaning for increasing $\mu$.

Historically heavy use has been made of relating the quark mass difference $m_{b}-m_{c}$ to the spin-averaged meson masses to reduce the number of parameters and to suppress ambiguities related to heavy mass definitions:

$$
m_{b}-m_{c}=\bar{M}_{B}-\bar{M}_{D}+\frac{\mu_{\pi}^{2}}{2}\left(\frac{1}{m_{c}}-\frac{1}{m_{b}}\right)+\frac{\rho_{D}^{3}-\bar{\rho}^{3}}{4}\left(\frac{1}{m_{c}^{2}}-\frac{1}{m_{b}^{2}}\right)+\mathcal{O}\left(1 / m_{Q}^{3}\right)
$$

where $\bar{M}_{B[D]} \equiv M_{B[D]} / 4+3 M_{B^{*}\left[D^{*}\right]} / 4$ denotes the spin averaged meson masses and $\bar{\rho}^{3}=$ $\rho_{\pi \pi}^{3}+\rho_{s}^{3}$ the sum of two positive (in the advocated scheme) nonlocal correlators (they are defined in Ref. [9]). Eq. (16) has become a widely used relation: for once one realizes that $\mu_{\pi}^{2}$ cannot differ from $0.45 \mathrm{GeV}^{2}$ by significantly more than $0.1 \mathrm{GeV}^{2}$, it leads to a reduced uncertainty in $m_{b}-m_{c}$. Having the same scheme and normalization point for both $m_{b}$ and $m_{c}$ has an advantage of making the heavy quark expansion relation between $m_{b}-m_{c}$ and $M_{B}-M_{D}$ more direct. If such constraints are not imposed, it is justified to use $\bar{m}_{c}\left(m_{c}\right)$ as well. We argue in Sect. 6 that it is safer not to invoke these relations.

\subsection{Operators of dimension 5 and 6}

The precise definition of higher-dimensional operators affects perturbative corrections to a lesser extent than the choice of the heavy quark mass scheme. Yet it is required to

\footnotetext{
${ }^{5}$ The perturbative contributions discussed here cannot give rise to spin-dependent effects contained in $\mu_{G}^{2}$
} 
make sense of assigning them a definite numerical value; once again the values have to be normalization-scale dependent. In particular this refers to the spin-singlet expectation values $\mu_{\pi}^{2}$ and $\rho_{D}^{3}$. They can be defined in the same physical way as the heavy quark masses; the formal definition is given, e.g. in [14]. Once given, it allows to sensibly extract their values; for instance, Ref. [14] concluded from the hyperfine mass splitting that

$$
\mu_{G}^{2}(1 \mathrm{GeV})=\left(0.35_{-.02}^{+.03}\right) \mathrm{GeV}^{2} .
$$

Kinetic expectation value $\mu_{\pi}^{2}$ a priori is less certain, however in this regularization scheme the inequality

$$
\mu_{\pi}^{2}(\mu) \geq \mu_{G}^{2}(\mu)
$$

holds for any normalization scale. With a number of experimental data strongly favoring $\mu_{\pi}^{2}(1 \mathrm{GeV}) \lesssim 0.5 \mathrm{GeV}^{2}$ a limited range is left allowed, and the estimate

$$
\left.\mu_{\pi}^{2}(1 \mathrm{GeV})\right)=(0.45 \pm 0.1) \mathrm{GeV}^{2}
$$

seems rather conservative. It is also supported by purely theoretical estimates including QCD sum rules [17]. ${ }^{6}$

A less precise estimate can be obtained for the Darwin expectation value, $\rho_{D}^{3}(1 \mathrm{GeV}) \simeq$ $(0.2 \pm 0.1) \mathrm{GeV}^{3}$, yet it must be positive. The spin-orbit average $\rho_{L S}^{3}$ is expected to be negative. It obeys the constraints

$$
-\rho_{L S}^{3} \leq \rho_{D}^{3}, \quad\left|\rho_{L S}^{3}\right| \leq 2 \rho_{D}^{3}
$$

Since the effect of this operator is typically strongly suppressed compared to the Darwin term, already these general bounds suffice for practical purposes.

A clarification is in order here. Strictly speaking, all the above bounds hold in the heavy quark limit, i.e. when $1 / m_{b}$ corrections in the expectation values are absent. It has been argued [14] that their shift from the $m_{b} \rightarrow \infty$ limit is negligible in $B$ meson. Not only it is governed by the parameter $1 / 2 m_{b}$, it should be additionally suppressed by virtue of the small excess of $\mu_{\pi}^{2}$ over $\mu_{G}^{2}$ manifesting proximity to the so-called BPS limit for the heavy meson ground state. Then the deviation of these hadronic parameters lies well below available precision, and can be neglected in practice.

\subsection{Operators with charm quarks}

As mentioned in the Executive Summary and the beginning of Sect. 2, four-quark operators containing a pair of charm and anti-charm quark fields necessarily arise in the OPE and their $B$ meson expectation values do not vanish exactly. These effects will be discussed in a forthcoming dedicated publication [11]. Here we give a brief introduction and state the salient conclusions of that analysis.

In discussing such effects in the OPE framework, it is essential to distinguish between an expansion in $1 / m_{b}$ (actually, $1 /\left(m_{b}-m_{c}\right)$ ) and in $1 / m_{c}$. For the sake of clarity we resort

\footnotetext{
${ }^{6}$ According to the FNAL group, lattice studies suggest so far a somewhat larger value [18].
} 
to a somewhat idealized scenario where the hierarchy between the two scales is stretched up compared to actual QCD.

To the leading third order in $1 / m_{b}$ there are four such operators of which two are relevant for $\Gamma_{\mathrm{sl}}(b \rightarrow c)$ :

$$
\begin{aligned}
H_{c}=\left\langle O_{1}^{c}\right\rangle & =-\frac{1}{2 M_{B}}\left\langle B\left|\bar{b} \gamma_{i}\left(1-\gamma_{5}\right) c \bar{c} \gamma^{i}\left(1-\gamma_{5}\right) b\right| B\right\rangle \\
F_{c}=\left\langle O_{2}^{c}\right\rangle & =-\frac{1}{2 M_{B}}\left\langle B\left|\bar{b} \frac{\lambda^{a}}{2} \gamma_{i}\left(1-\gamma_{5}\right) c \bar{c} \frac{\lambda^{a}}{2} \gamma^{i}\left(1-\gamma_{5}\right) b\right| B\right\rangle .
\end{aligned}
$$

( $F_{c}$ is the tree-level counterpart of $\tilde{H}_{c}$ in Eq. (8)). To higher orders in $1 / m_{b}$ there are additional operators with derivatives acting on the quark fields. They can be reasonably neglected in the total width. For general purposes it is convenient to consider the extended set of four such operators

$$
\begin{array}{ll}
O_{V}^{s}=\bar{b} b \bar{c} \gamma_{0} c & O_{A}^{s}=\bar{b} \vec{\sigma} b \bar{c} \vec{\gamma} \gamma_{5} c \\
O_{V}^{o}=\bar{b} \frac{\lambda^{a}}{2} b \bar{c} \frac{\lambda^{a}}{2} \gamma_{0} c & O_{A}^{o}=\bar{b} \frac{\lambda^{a}}{2} \vec{\sigma} b \bar{c} \frac{\lambda^{a}}{2} \vec{\gamma} \gamma_{5} c
\end{array}
$$

which define the expectation values $H_{c}$ and $F_{c}$ by virtue of Fierz transformations:

$$
\begin{aligned}
O_{1}^{c} & =-\frac{3}{2 N_{c}} O_{V}^{s}+\frac{1}{2 N_{c}} O_{A}^{s}-3 O_{V}^{o}+O_{A}^{o} \\
O_{2}^{c} & =-\frac{3}{4}\left(1-\frac{1}{N_{c}^{2}}\right) O_{V}^{s}+\frac{1}{4}\left(1-\frac{1}{N_{c}^{2}}\right) O_{A}^{s}+\frac{3}{2 N_{c}} O_{V}^{o}-\frac{1}{2 N_{c}} O_{A}^{o} .
\end{aligned}
$$

The indices $V, A$ and $s, o$ correspond to products of vector or axial, and color-singlet or -octet flavor-diagonal $\bar{b} b$ and $\bar{c} c$ currents. Below we shall mainly refer to the contributions related to $H_{c}$ as most relevant for $\Gamma_{\mathrm{sl}}(b \rightarrow c)$.

The existence of such an effect can be understood since this expectation value simply states that the propagation of the decay quark inside $B$ meson is not totally perturbative. It is related to local expectation values because we consider the inclusive width coming from $c$ quark momenta much smaller than the energy release where the momentum of the lepton pair does not differs much from $q_{\mu}=\left(E_{r}, 0,0,0\right)$, and we expand in this difference [19]. This inclusive probability for transitions to charm quarks is affected by nonperturbative dynamics to a lesser degree than for decays to light quarks; yet it is evident that it should be present at some level. Its magnitude can be qualified and estimated [11]. The relation of these effects to the "Intrinsic Charm" in $B$ mesons is discussed there.

The charm quark propagator undergoes nonperturbative effects even when it is hard or has large energy or momentum. These effects, however are accounted for in the standard OPE; to the leading order they are given in terms of $\mu_{\pi}^{2}$ and $\mu_{G}^{2}$ as exemplified by the sum rules considered in Ref. [9]. The effect of the Darwin term comes from both hard and soft charm configurations. Hence, in the Wilsonian approach a part of the effect of the Darwin operator in Eq. (8) actually represents the contribution $H_{c}$ of the four-quark charm operator. This part belonging to $\rho_{D}^{3}$ depends on the choice of the normalization 
point $\mu$ and vanishes if $\mu$ is taken well below $m_{c}$ - this option is possible only for a heavy quark.

If the charm field can be considered as heavy in the scale of typical hadronic masses $\mu_{\text {hadr }}$, one can apply heavy quark expansion to these nonperturbative expectation values. It proceeds, however, in $1 / m_{c}$ and not in $1 / m_{b}$ :

$$
\left\langle O_{j}^{c}\right\rangle=\sum_{k} C_{j k} \frac{1}{2 M_{B}} \frac{\left\langle B\left|\bar{b} O_{k} b(0)\right| B\right\rangle}{m_{c}^{d_{k}-3}} .
$$

Similar to the OPE for inclusive widths, it includes only local $\bar{b} \ldots b$ operators which, however, now involve only light (gluon and $u, d, s$ quark) fields. In the above equation $d_{k}$ denotes the dimension of the light field operator $O_{k}$. Therefore, without introducing the explicit operators with charm, there exists a chain of higher-order power terms scaling like $\frac{\Lambda_{Q C D}^{n}}{m_{b}^{3} m_{c}^{n-3}}$ rather than $\frac{\Lambda_{Q C D}^{n}}{m_{b}^{n}}$. It is instructive to keep in mind that the large coefficient of the Darwin operator is associated with the lowest term in this subseries corresponding to $n=3$ which gives rise to the $\ln \frac{1}{m_{c}}$ enhancement.

The $1 / m_{c}$ expansion of the charmed expectation values thus requires classifying possible operators $O_{k}$ and computing the coefficients $C_{j k} \cdot{ }^{7}$ It is shown [11] that at the tree level there are no contributions to order $1 / m_{c}$, while $1 / m_{c}^{2}$ effects are driven by a few operators, depending on the particular color and Lorentz structure. ${ }^{8}$ The dominant in $N_{c}$ structure for semileptonic decay is color octet, see Eq. (23), for which $1 / m_{c}^{2}$ effects are present for both vector and axial currents.

It is interesting, however, that absence of $1 / m_{c}$ nonperturbative charm loop effects can be vitiated once perturbative corrections are included [11]. There are two spin-singlet operators $O_{E^{2}}$ and $O_{E E}$ and one spin-dependent $O_{E \times E}$ operator which emerge already to order $\alpha_{s}\left(m_{c}\right)$ from the dominant (in semileptonic decays) color-octet operators, vector and axial, respectively:

$$
\begin{aligned}
O_{E^{2}} & =\frac{1}{16 \pi^{2}} \bar{b} \operatorname{Tr} \vec{E}^{2} b(0)=\frac{1}{16 \pi^{2}} \bar{b} \operatorname{Tr}\left(\pi_{k} \pi_{0} \pi_{0} \pi_{k}\right) b \\
O_{E E} & =\frac{1}{16 \pi^{2}} \bar{b}\left(1-\frac{1}{N_{c}} \operatorname{Tr}\right) \vec{E} \cdot \vec{E} b(0)=\frac{1}{16 \pi^{2}} \bar{b}\left(1-\frac{1}{N_{c}} \operatorname{Tr}\right)\left(\pi_{k} \pi_{0} \pi_{0} \pi_{k}\right) b \\
O_{E \times E} & =\frac{1}{16 \pi^{2}} i \vec{b} \vec{\sigma} \cdot \vec{E} \times \vec{E} b(0)=-\frac{1}{16 \pi^{2}} i \epsilon^{\mu \nu \rho \lambda} \bar{b} \gamma_{\mu} \gamma_{5} \pi_{\nu} \pi_{\rho} \pi_{0} \pi_{\lambda} b
\end{aligned}
$$

where $\vec{E}$ is the non-Abelian chromoelectric field strength (it includes coupling $g_{s}$ ), and $\pi_{\mu}$ are the nonrelativistic energy-momentum operators. An example of the Lorentz-invariant operator yielding such spin-dependent interaction is

$$
\frac{1}{16 \pi^{2} m_{b}} \epsilon^{\mu \nu \rho \lambda} \bar{b} \gamma_{\mu} \gamma_{5} D_{\nu} D_{\rho} D_{\alpha} D_{\lambda} D^{\alpha} b
$$

\footnotetext{
${ }^{7}$ Alternatively, one can integrate the sum rules for the corresponding combinations of the zeroth moments (for the leading- $m_{b}$ operators) of the heavy quark structure functions studied in [9], over $\vec{q}$. This would require, however extending the expansion at least to two more orders in $1 / m_{c}$.

${ }^{8}$ Say, no contribution for the color-straight operator with vector currents, one for the product of color-straight axial currents, etc.
} 
with $D_{\mu}$ denoting the full-QCD covariant derivatives. Such operators come with coefficient $\propto \alpha_{s}\left(m_{c}\right) / \pi$ and are not expected to dominate for actual charm quark. They would represent the leading effect in the true heavy charm limit. Physically $1 / m_{c}$ corrections can describe interference between the (gluon-dressed) parton decay amplitude and internal $c \bar{c}$ component of the meson, see Fig. 1 similar to interference discussed in detail in Ref. [20]. Soft fields shape the internal charm component of wavefunction, while short-distance effects here are characterized by virtuality starting at $m_{c}$ and governed by $\alpha_{s}\left(m_{c}\right)$. To even higher order in $\alpha_{s}\left(m_{c}\right)$, and/or including gluons with momentum scaling as $m_{b}$ more operators become possible.

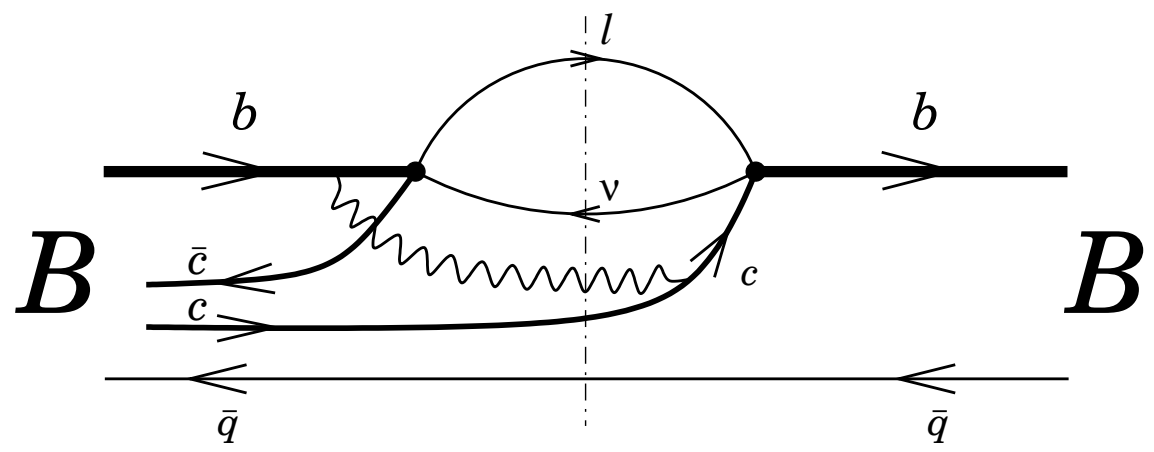

Figure 1: Example of the interference contribution to the decay width suppressed by a single power of $1 / m_{c}$. Shown gluon is hard. All cuts should be included to maintain the correct scaling.

The effects of intrinsic charm in the context of DIS on nucleons were thoroughly investigated in Ref. [21], though without perturbative effects. The relevant charm operators are quark bilinears in this case, yielding quite different pattern of the power expansion. Including hard perturbative corrections can change leading power in $1 / m_{c}$ for heavy quark decays, as noted above.

The practical OPE Eq. (24) in the form of an expansion in $\Lambda_{\mathrm{QCD}} / m_{c}$ is only asymptotic; the charm expectation values contain also "exponential" pieces like $e^{-2 m_{c} / \mu_{\mathrm{hadr}}}$. Various considerations lead us to expect that for actual charm with $m_{c} \simeq 1.2 \mathrm{GeV}$ these exponential terms can be, if not dominant, at least not too far suppressed compared to the leading powerlike effects. These contributions would represent the clearest and unambiguous example of genuinely independent effects associated with nonperturbative corrections for charm quarks in $B$ mesons.

All these contributions, which are suppressed by powers of $1 / m_{c}$ and therefore could be sizeable thus posing a danger to the $1 / m_{Q}$ expansion, just reflect the existence of operators in the OPE of the width containing pairs of $c$ and $\bar{c}$ fields, namely $O_{j}^{c}$ (and their higher-dimensional analogies). Their impact is best analyzed directly through the $B$ expectation values of $O_{j}^{c}$. The size of these nonperturbative expectation values is however not very certain. For $\Gamma_{\mathrm{sl}}(b \rightarrow c)$ there is a single relevant operator, Eq. (11). The size of its expectation value $\left|H_{c}\right|$ is estimated [11]

$$
\left|H_{c}\right| \lesssim 0.005 \mathrm{GeV}^{3}
$$


which translates into a possible contribution to the width up to 1 to $1.5 \%$. This looks reasonable if we compare it with the effect of the Darwin expectation value itself which, according to our master equations is estimated to be about 3\%. A fraction of the Darwin term effect, on the other hand, represents the leading, $1 / m_{c}^{0}$ piece of the four-quark operators with charm.

An alternative way to arrive at a similar numerical estimate is calibrating them with the size of nonfactorizable contribution of four-quark operators $\bar{b} b \bar{q} q$ with light quarks $q$, which would enter the total semileptonic $b \rightarrow u$ width. The four-quark operators with non-valence light quark were estimated in Ref. [22] to affect the width at a couple percent level, employing the information about $D$ meson widths. Assuming that the expectation values with the charm field replacing light non-valence quarks exhibit extra suppression due to the charm mass by a factor of four, and accounting for the difference in the phase space in the two decays, we end up with an estimate somewhat below the one percent level in $\Gamma_{\mathrm{sl}}(B)$.

\subsubsection{Experimental constraints}

A more robust approach is to constrain the charm-related expectation values directly from experiment. Available studies of the semileptonic distributions already yield some more indirect bounds on such effects, however the limits fall quite short of the scale expected theoretically. One reason behind this insensitivity is transparent: they manifest themselves in the same way as the soft part of the Darwin term, and therefore to some extent can be absorbed by redefining the effective value of $\rho_{D}^{3}$. In particular, for sufficiently inclusive characteristics replacing

$$
d \rho_{D}^{3} \longrightarrow d \rho_{D}^{3}+32 \pi^{2}(1-\sqrt{r})^{2} H_{c}
$$

may account for the bulk of the effect, and the actual difference in the effect of Darwin operator and the charm-related nonperturbative four-quark expectation value $H_{c}$ will be revealed at a suppressed level only in more subtle quantities, say in the dependence of the hadronic distributions on lepton energy.

There can be various physical manifestations of the nonperturbative effects associated with moderately short-distance charm quarks in non-charm beauty hadrons. A number of effects have been discussed in connection to the "intrinsic charm" hypothesis [23]; it is not always easy to disentangle them from other underlying effects, however.

Here we are concerned with a more concrete aspect, namely the way such effects may manifest themselves in the inclusive semileptonic decays via local $b$-quark operators with charm, Eqs. (22). The search for an unambiguous manifestation is complicated by the expected percent scale in $\Gamma_{\mathrm{sl}}(b \rightarrow c)$. Yet even any higher direct upper bound would constitute a very valuable information complementary to theoretical estimates.

In the heavy quark expansion these four-quark operators would most directly show up as $\delta$-like contributions near the quasi-two-body kinematics $b \rightarrow$ slow $c+\ell \nu$, which naively 
corresponds to such leading terms in the OPE, with

$$
\begin{aligned}
\frac{1}{\Gamma_{\mathrm{sl}}} \frac{\mathrm{d} \Gamma_{c c}}{\mathrm{~d} q^{2}} & \simeq \frac{16 \pi^{2}}{M_{B}-M_{D^{*}}} \frac{H_{c}}{m_{b}^{3}} \delta\left(\sqrt{q^{2}}-\left(M_{B}-M_{D^{*}}\right)\right) \\
\frac{1}{\Gamma_{\mathrm{sl}}} \frac{\mathrm{d} \Gamma_{c c}}{\mathrm{~d} E_{X}} & \simeq 32 \pi^{2} \frac{H_{c}}{m_{b}^{3}} \delta\left(E_{X}-\left(M_{B}-M_{D^{*}}\right)\right), \\
\frac{1}{\Gamma_{\mathrm{sl}}} \frac{\mathrm{d} \Gamma_{c c}}{\mathrm{~d} E_{\ell}} & \simeq 32 \pi^{2} \frac{H_{c}}{m_{b}^{3}} \delta\left(E_{\ell}-\frac{M_{B}-M_{D^{*}}}{2}\right)
\end{aligned}
$$

etc. Placed in the narrow slice of the whole kinematic domain, these effects are locally enhanced and potentially detectable in differential distributions, much in the same way as was suggested for nonfactorizable effects in $\bar{b} b \bar{q} q$-type operators with light quarks in the lepton spectra [19], or $M_{X}^{2}$ or $E_{X}$ distributions [24].

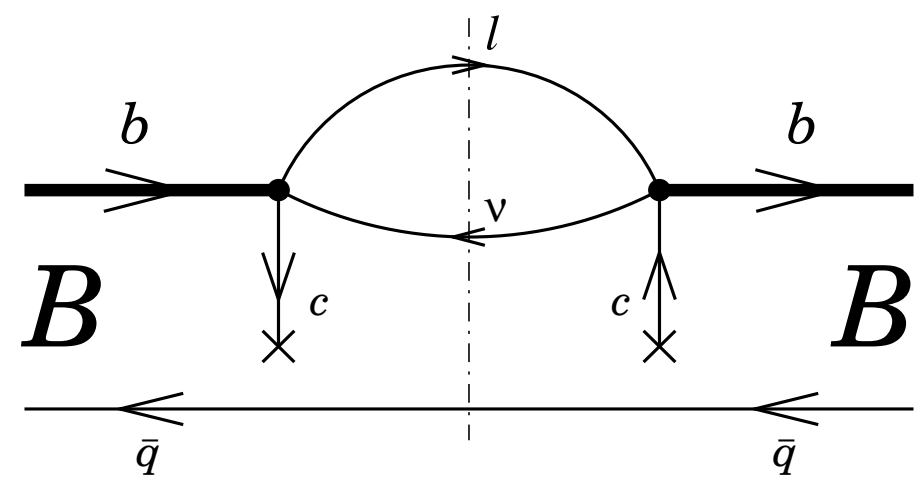

Figure 2: Nonperturbative charm-field effects in the OPE for the inclusive decays. With charm fields being low-momentum, the kinematics is close to two-body for leptons, with $\sqrt{q^{2}} \simeq m_{b}-m_{c}$.

There are also similar operators with derivatives. In the total widths they are suppressed by additional powers of $1 / m_{b}$. They lead to smearing the above quasi-two-body peaks over the domain of typical width $\sim \Lambda_{\mathrm{QCD}}$. The smearing in practice can stretch over a sizable fraction of the allowed kinematic range for actual $m_{b} .{ }^{9}$ It should be noted that the charm operators can lead to both enhancement or depletion of the decay rates, depending on the sign of the expectation values.

The above pattern suggests the strategy of studying the integral over the particular low- $E_{X}$ kinematic domain and comparing it with the expectations based on the parameters extracted from the total moments. It turns out that the overall integrated hadronic energy $\left\langle E_{X}\right\rangle$ and the invariant mass $\left\langle M_{X}^{2}\right\rangle$ are theoretically expected to depend on practically one and the same combination of parameters $m_{b}-0.67 m_{c}+0.1 \mu_{\pi}^{2}-0.25 \rho_{D}^{3}$. Comparing the two averages experimentally would then be a cross check of possible additional effects, including charm expectation values.

\footnotetext{
${ }^{9}$ Perturbative corrections associated with the anomalous dimensions of the leading operators yield slower decreasing tails as discussed in Ref. [19]; yet in practice this perturbative smearing should hardly be relevant.
} 
It seems to us that the available accuracy in the fully integrated moments (with respect to $E_{\ell}, M_{X}$ or $q^{2}$ ) does not allow at present to constrain the expectation values, in particular $H_{c}$ with the precision even nearly matching the theoretical expectations Eq. (26). An interesting possibility is to study the partially integrated over $E_{X}$ double differential distribution

$$
\Delta\left(\vec{q}^{2} ; M\right)=\frac{1}{\Gamma_{\mathrm{sl}}} \int_{M_{D}^{2}}^{M_{D^{*}+M}} \mathrm{~d} M_{X}^{2} \frac{\mathrm{d}^{2} \Gamma_{\mathrm{sl}}}{\mathrm{d} M_{X}^{2} \mathrm{~d} \vec{q}^{2}} .
$$

For the four-fermion operators it is expected to exhibit a more or less flat contribution above a hadronic scale $M_{0}$ for moderate $\vec{q}^{2}$,

$$
\int \mathrm{d} \vec{q}^{2} \Delta\left(\vec{q}^{2}\right) \simeq 32 \pi^{2} \frac{H_{c}}{m_{b}^{3}}
$$

which however fades away with increase of $\vec{q}^{2}$. In this scenario the effect can originate from a limited range of $M_{X}$ below a hadronic mass $M_{D^{*}}+M_{0}$ with $M_{0}$ expected to be around $m_{c}$ and/or $\mu_{\text {hadr }} \approx 1 \mathrm{GeV}$.

The most precision data available now are from CLEO lepton moments measured with a lower cut $1.5 \mathrm{GeV}$ [25]. However, the average lepton energy in this interval is $\left\langle E_{\ell}\right\rangle \simeq 1.73 \mathrm{GeV}$ which is very close to the expected location of the discussed contributions

$$
E_{\ell}^{c c} \approx \frac{M_{B}-M_{D}^{*}}{2} \simeq 1.64 \mathrm{GeV}
$$

This strongly suppresses the sensitivity of these moments to $H_{c}$. At the same time, this physical example illustrates the fact that the integrated widths with cuts are far more vulnerable to higher-order effects: the relative sensitivity of just the rate with $E_{\ell}>$ $1.5 \mathrm{GeV}$ to $H_{c}$ can naturally be three times higher than of $\Gamma_{\mathrm{sl}}^{\text {tot }}(b \rightarrow c)$ simply because such a partial width comprises only about $30 \%$ of the total rate, and impact on the CLEO's $R_{0}$ can be even more significant. Yet we think it may be premature to attribute some apparent discrepancy between, say, measured values of $R_{0}$ and $R_{1}$ to effects of charm operators, in view of insufficient control over usual higher-dimension operators for the widths with such a cut [26].

It is also worth emphasizing that at a high enough cut on $E_{\ell}$ the operators describing the addressed nonperturbative effects cease to remain local (in space). Whether the cut at $1.5 \mathrm{GeV}$ is safe in this respect, must yet be understood.

\subsection{Heavy quark operators of $D \geq 7$}

The important question about the achievable accuracy of the OPE for $\Gamma_{\mathrm{sl}}(b \rightarrow c)$ is the magnitude of higher-order power corrections beyond $1 / m_{b}^{3}$ terms. Their number proliferates, and it might look hopeless to get a meaningful answer. Nevertheless, we shall argue that the effect of the higher-dimensional operators while applying the proper Wilsonian procedure should be suppressed and not exceed the percent level. One also needs to qualitatively understand the hierarchy of the computed corrections through order $1 / m_{b}^{3}$. This is discussed later in this section. 
To get the way around apparent complexity of higher-order corrections, we use a few basic ideas. A few different physical momentum scales enter the problem even if one abstracts from perturbative corrections generally messing up physics at different distances. Namely, a number of corrections are driven solely by the scale $1 / 2 m_{b}$ - among those are preasymptotic corrections to the expectation values of nonperturbative operators over the actual $B$ meson state. It is clear that these terms among higher-order operators can be neglected at once.

The second largest scale specific to the inclusive width is the energy release $m_{b}-m_{c} \simeq$ $3.5 \mathrm{GeV}$. Since it still is significant, the higher-dimension operators controlled by such an expansion parameter yield the contribution far below the percent level as is illustrated by the $L S$ term, unless their effect is particularly enhanced. There are two reasons for a possible enhancement of the coefficient functions. One is the "infrared instability" when $m_{c} / m_{b} \rightarrow 0$. It was discussed in Sect. 3.3, and they are taken care of by introducing the explicit expectation values $H_{c}, \tilde{H}_{c}$ of the four-quark operators with charm in Eq. (8). Indeed, infrared sensitivity is possible only for soft $c$ quark line, with the lepton pair carrying the momentum close to $m_{b}-m_{c}$. This effectively contracts the two weak vertices in space and time yielding local four-fermion operator $\bar{b} \Gamma c \bar{c} \Gamma b$.

The second reason discussed in detail in Ref. [8] is due to presence of the large parameter $N=5$ describing the power $N$ with which heavy quark masses enter the total width, $\Gamma \propto\left(m_{b}, m_{b}-m_{c}\right)^{5}$. Therefore, to find the way through the mace of higher-order power corrections, we will use the classification of the coefficients over this parameter. ${ }^{10}$ Namely, we can require an extra power of the hadronic scale $\mu_{\text {hadr }} / m_{b}$ in the nonperturbative corrections to be accompanied by the maximal power of $N$.

The $1 / N$ classification is quite transparent and yields a number of useful facts. As pointed out in Ref. [8], large $N$ pushes one to the so-called Extended SV regime, since large $N$ places most of the weight on maximal $q^{2}$ of the lepton pair. It is also evident that this dominance is further enhanced for higher-dimensional operators: the leading contribution comes from multiple differentiation of the $c$ quark propagator with respect to $m_{c}^{2}$, which pushes it further and further into the infrared. In this respect the effect is the same as for the first mechanism, infrared enhancement, and in the same way the largest pieces are absorbed into the explicit charmed expectation values. The upshot of this approach is two-fold:

- The dominant operators are spin-singlet like the kinetic and Darwin terms, as opposed to the spin-dependent chromomagnetic or $L S$ operators. These are among those which determine higher moments of the light-cone distribution function. The lightcone set of operators would dominate if $m_{b}$ were really much greater than $m_{c}$ so that $m_{b} / N>m_{c}$, the case where normalization point $\mu$ for the four-fermion operators with charm could be taken well above $m_{c}$.

- In the realistic case, the leading ones among these spin-singlet operators are those containing more time derivatives; extra space derivatives generally yield subleading in

\footnotetext{
${ }^{10}$ The increase in the Wilson coefficients should be distinguished from the expected factorial growth of the matrix elements of the operators compared to the dimensional estimate $\mu_{\text {hadr }}^{k}$; the latter is related to violation of local duality $[27,28]$ and will be addressed separately.
} 
$N$ effects. Since the left-most and right-most covariant derivatives in $\langle\bar{b} D \ldots D b\rangle$ must be space-like for the heavy $b$ quark, it is actually the SV-type operators determining the SV heavy quark distribution function that dominate the higher-order power corrections. This is in a clear accord with the onset of the Extended SV regime mentioned above.

It is worth noting that the dominance of the SV-like operators makes the estimates more conclusive. They can be fully defined with explicit normalization-point dependence in the same way as the operators through $D=6$, see, e.g. $[29,7,14]$ :

$$
\left\langle\bar{b} i D_{k}\left(i D_{0}\right)^{n} i D_{k} b\right\rangle_{\mu}=3 \sum_{\epsilon<\mu}\left[2\left(\epsilon_{3 / 2}^{(l)}\right)^{n+2}\left|\tau_{3 / 2}^{(l)}\right|^{2}+\left(\epsilon_{1 / 2}^{(m)}\right)^{n+2}\left|\tau_{1 / 2}^{(m)}\right|^{2}\right] .
$$

This eliminates the notorious problem with fixing the ad hoc factors which often can be arbitrarily reshuffled between the operators and their Wilson coefficients, obscuring numerical estimates. For instance, the parameter $\lambda_{2}$ used in some papers historically was defined with the factor $1 / 3$ of the actual size of the physical chromomagnetic operator in $B$, and HQET's $\lambda_{1}$ was laxly defined as minus the expectation value of the positive operator.

The chain of SV expectation values has a definite sign. In addition, their scaling is given by a physical mass, the energy of the excited $P$-wave states at which one observes the onset of the perturbative regime. The recent data on the $M_{X}$ spectrum in semileptonic $B$ decays $[30,31]$ suggest that this scale is near the expected $1 \mathrm{GeV}$. It is clear that the magnitude of the ratio of this mass to the energy release $m_{b}-m_{c}$ would govern the rate of convergence of power expansion. For the SV regime, for example, this is explicit in the analysis of the Orsay group [32]. The detailed study of the $M_{X}$ spectrum in semileptonic decays will allow even some more quantitative bounds on the leading higher-order heavy quark operators.

The analysis of possible higher-order effects conducted along these lines suggests that the effect of higher-order operators with $D \geq 7$ constitutes at most a fraction of percent in $\Gamma_{\mathrm{sl}}(b \rightarrow c)$, at least if the four-quark operators with charm field are properly introduced to take care of the infrared contributions. It is worth commenting in this respect on the numerical pattern in low-order power corrections, which is not always properly interpreted and may lead to unjustified suspicions about convergence.

According to Eq. (1), the first few power corrections amount, in fractional units to

$$
\delta_{\mu_{\pi}^{2}} \simeq-0.012, \quad \delta_{\mu_{G}^{2}} \simeq-0.035, \quad \delta_{\rho_{D}^{3}} \simeq-0.035, \quad \delta_{\rho_{L S}^{3}} \simeq-0.003 .
$$

The values $\mu_{\pi}^{2}=0.4 \mathrm{GeV}^{2}, \mu_{G}^{2}=0.35 \mathrm{GeV}^{2}, \rho_{D}^{3}=0.2 \mathrm{GeV}^{3}$ and $\rho_{L S}^{3}=-0.15 \mathrm{GeV}^{3}$ have been used. The significant contribution of the Darwin term compared to the second-order corrections, and in particular, to the kinetic operator, may raise some concern. In fact, applying the above theoretical perspective one can realize that the Darwin operator has the expected, normal size.

First of all, as detailed earlier, a significant part of the Darwin expectation value actually represents the contribution of the four-quark charm operator $H_{c}$ in Eq. (11) if it is understood in the Wilsonian sense normalized at the appropriate scale, say at 
$\mu \simeq m_{c}+1 \mathrm{GeV}$. The remaining part is then noticeably smaller than the estimate of Eq. (32).

It is more important to appreciate the following fact: the effect of the kinetic and chromomagnetic operators is actually essentially suppressed compared to a priori expectations based on the scale arguments. These are nontrivial dynamic results of the OPE which cannot be accounted for by simple dimensional considerations.

The most obvious example comes from $1 / m_{b}$ effects. Dimensional estimates would yield

$$
\delta_{1 / m_{b}} \simeq 5 \frac{\bar{\Lambda}}{m_{b}},
$$

the result typically exhibited by naive quark model calculations. Such a correction would actually belong to the SV family in Eq. (31): according to Voloshin's "optical" sum rule $\frac{3}{2} \bar{\Lambda}$ amounts to the expectation value with $n=-1$. Such corrections physically exits. However, the OPE ensures that in QCD they exactly cancel between the binding effects in the initial $B$ state and hadronization corrections in the final state. Clearly one would not interpret the fact that $1 / m_{b}^{2}$ corrections to total widths are infinitely larger than the absent $1 / m_{b}$ effects, as a non-convergence of the power series!

It is even more interesting that a similar cancellation, in a sense holds even to second order in $1 / m_{b}$. Naively one would expect the kinetic operator to appear with the coefficient scaling like $5 \cdot 4 / 2 \gg 1$, and to have powers of $m_{b}-m_{c}$ rather than $m_{b}$ in denominator. However, the OPE once again ensures that such an effect is totally absent from the inclusive width if strong interactions are described by a gauge theory like QCD. ${ }^{11}$ The only effect of the kinetic operator emerges as the $1 / m_{b}^{2}$ correction to the expectation value $\langle B|\bar{b} b| B\rangle[10,34,9]$. This makes evident why its effect is numerically suppressed. We can safely discard such contributions for higher-order operators, which has been already assumed in formulating the rules of the analysis earlier in this Section.

The coefficient of the chromomagnetic operator does not vanish. Yet it is still partially suppressed, which can be explicitly seen computing its effect when employing $N=5$ as a free parameter: it scales like $N$, but not $N^{2}$. This is related to the spin-nonsinglet structure of the chromomagnetic operator - it does not belong to the SV family of operators of Eq. (31) being an antisymmetric combination of the spacelike derivatives. This partial suppression is also reflected in the dependence of its coefficient in the width: compared to the parton width it is enhanced by a single power of $m_{b} /\left(m_{b}-m_{c}\right)$.

A similar partial suppression is present for all higher-order spin-nonsinglet operators, which is precisely illustrated by the $L S$ contribution. It has a coefficient scaling like that of $\mu_{G}^{2}$, and its numerical contribution is safely at a sub-percent level.

For the Darwin operator, however, the cancellations 'accidentally' enforced on the lower-dimension SV parameters in the OPE do not hold anymore, and its coefficient has 'normal' or expected magnitude. Its numerical impact on the total width is on general grounds expected to be in a few percent range, assuming the normal scaling

\footnotetext{
${ }^{11}$ Even refined parton-based models typically predict a large positive effect of the kinetic expectation value; see, for example the light-cone based approach of Ref. [33] yielding the coefficient $+35 / 6$.
} 
of the expectation values following from the family of the SV sum rules $[1,7]$. The effect of higher-order operators then should not exceed the scale of a half percent.

To summarize, we expect, and have argued that the contribution of heavy quark operators with $D \geq 7$ can give only a sub-\% effect on $\Gamma_{\mathrm{sl}}(b \rightarrow c)$ provided the four-fermion operators with charm field in the Wilsonian OPE have been properly incorporated.

\section{Perturbative Contributions}

Once the perturbative effects in the quark masses and higher-dimensional operators have been properly incorporated, one can turn to the radiative corrections to the Wilson coefficients. In practice, high accuracy is required for the leading (parton) operator coefficient denoted as $1+A_{3}^{\text {pert }}$ in Eq. (8), and we routinely refer to it as the perturbative correction $A^{\text {pert }}$, see Eq. (1).

It has become customary to express perturbative corrections as series in the $\overline{\mathrm{MS}}$ coupling evaluated at $m_{b}$. Yet the scales typical for the radiative corrections are notably lower than $m_{b}$. By evaluating the lowest order term in $\alpha_{s}^{\overline{\mathrm{MS}}}\left(m_{b}\right)$ one generally underestimates $A^{\text {pert }}$ and forces the $\alpha_{s}^{2}$ and higher terms to possess large coefficients. If, however, at least the second order BLM contributions are known, then one can express the findings in terms of $\alpha_{s}^{\overline{\mathrm{MS}}}\left(m_{b}\right)$ without raising numerical havoc.

It should be noted that using the Wilsonian prescription with an appropriate hard factorization scale $\mu$ significantly improves convergence of the perturbative series and makes the above problem less acute. This is illustrated by Fig. 3 showing the contribution of different momentum scales in the total width, with and without separation of shortand long-distance effects. ${ }^{12}$ The effect of removing the infrared domain is self-manifest. Moreover, it is evident that a too significant part of the perturbative corrections with pole masses comes from gluon momenta below $500 \mathrm{MeV}$, which casts serious doubts on reliability of the numerical results in this scheme.

\subsubsection{BLM summation}

Computing the leading-order $\mathcal{O}\left(\alpha_{s}\right)$ contribution does not tell us at which scale the coupling has to be evaluated; that is fixed only by terms of higher order in $\alpha_{s}$. The (extended) BLM prescription $[35,36,37]$ is based on the conjecture that the bulk of higher order terms can be incorporated by replacing the fixed lowest order $\alpha_{s}$ by the scale-dependent $\alpha_{s}\left(k^{2}\right)$ where $k$ denotes the momentum flowing through the gluon line, cf. Fig. 1. The running of $\alpha_{s}$ is determined by the first coefficient of the Gell-Mann-Low beta function: $\beta_{0}=\frac{11}{3} N_{c}-\frac{2}{3} n_{f} \simeq 9$. The resulting expansion is of the type $\alpha_{s}\left(1+\sum_{n=1}^{\infty} c_{n}\left(\beta_{0} \alpha_{s}\right)^{n}\right)$ with $n=1,2, \ldots$ representing the second-order, third- etc. BLM correction. The BLM prescription amounts to the assumption that the perturbative terms left out are much

\footnotetext{
${ }^{12}$ We show the distribution from Ref. [5] which did not incorporate $1 / m_{b}^{3}$ subtraction corresponding to Darwin operator.
} 


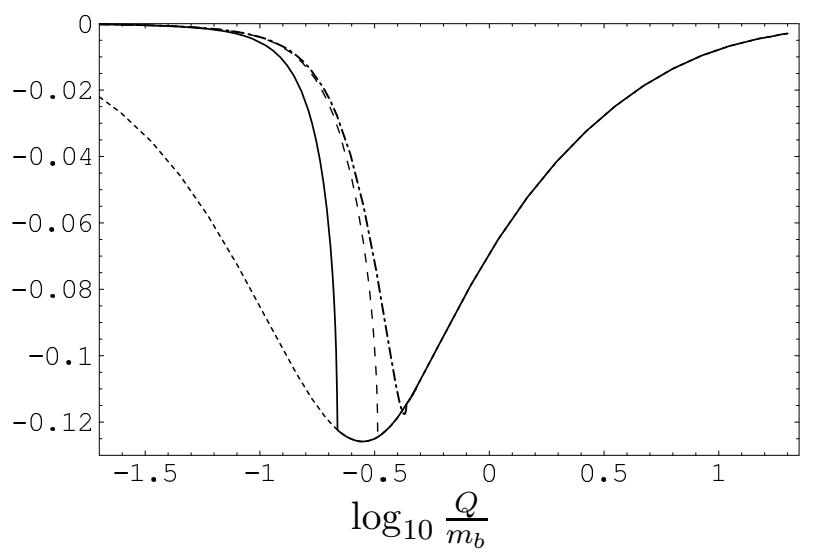

Figure 3: Gluon momentum scale distribution in $\Gamma_{\mathrm{sl}}(b \rightarrow c)$. Solid, dashed and dot-dashed lines correspond to $\mu=1 \mathrm{GeV}, 1.5 \mathrm{GeV}$ and $2 \mathrm{GeV}$, respectively; lighter short-dashed line illustrates the case of $\mu=0$ (pole masses). The area under each curve gives the first-order perturbative coefficient.

smaller since not enhanced by the large value of $\beta_{0}$. A dedicated recent discussion can be found in Ref. [38].

This ansatz has turned out to be intriguingly successful in several cases. Often it is used in a simplified form by considering only the first nontrivial BLM correction - this constitutes the essence of the BLM scale fixing for $\alpha_{s}$ [35]. However in applying this properly to semileptonic $B$ decays one has to be aware of some specific complexities:

- There are effectively two 'running' couplings in the problem, namely $\alpha_{s}$ and $m_{b}$. There is no reason why they should be controlled by the same scale - on the contrary, one expects them to be different. Moreover, the standard BLM scale fixing procedure is not defined - and rather often becomes meaningless - in the case of more than one coupling. Indeed, varying the normalization scale for masses the BLM scale for $\alpha_{s}$ varies in the whole range from zero to infinity.

- As stated before good judgment has to be exercised in choosing the proper mass construction. The pole mass with its intrinsic infrared uncertainty $\sim \mathcal{O}\left(\Lambda_{\mathrm{QCD}}\right)$ is ill-suited in this context. Using the BLM improvement only aggravates this problem.

The analytic expressions for the perturbative corrections and the way to implement the Wilsonian cutoff are described in Appendices A.2 and A.3. They also give numerical values.

\subsubsection{Non-BLM contributions}

The non-BLM terms are expected to be small. They are known in the analytic form neglecting the charm mass (the case of $\Gamma_{\mathrm{sl}}(b \rightarrow u)$ ) [39]. In $b \rightarrow c$ a numerical evaluation exists. The authors of Ref. [40] have computed $\mathcal{O}\left(\alpha_{s}^{2}\right)$ corrections to $b \rightarrow c \ell \nu$ at the three 
different kinematical points $q^{2}=0, m_{c}^{2}$ and $\left(m_{b}-m_{c}\right)^{2}$, where $\sqrt{q^{2}}$ denotes the mass of the lepton pair. From these three values one interpolates over the full $q^{2}$ range. The nonBLM corrections indeed turn out to be small for the low-scale masses, which is a highly welcomed result: the consistent procedure to fix the scale of $\alpha_{s}$ in the non-BLM terms is not known unless third-order corrections beyond simple BLM are available. This would yield significant numerical uncertainty if the non-BLM coefficients were not suppressed.

\subsection{Overall perturbative correction}

It is gratifying that the perturbative corrections to $\Gamma_{\mathrm{sl}}(b \rightarrow c)$ with the short-distance low-scale masses show good convergence [41]. Let us define

$$
z_{0}(r) A^{\text {pert }}(r ; \mu)=z_{0}(r)+a_{1} \frac{\alpha_{s}}{\pi}+a_{2}\left(\frac{\alpha_{s}}{\pi}\right)^{2}+a_{3}\left(\frac{\alpha_{s}}{\pi}\right)^{3}+\ldots
$$

assuming the standard choice of the $\overline{\mathrm{MS}}$ coupling $\alpha_{s}(M)$ normalized at $M=m_{b}$. As an example, at $\mu=1 \mathrm{GeV}, m_{b}=4.6 \mathrm{GeV}$ and $m_{c}=1.15 \mathrm{GeV}$ we have

$$
\begin{array}{rlrl}
a_{1} & \simeq-0.94 z_{0}(r), \quad a_{2}^{\text {non-BLM }} & \simeq-1.0 z_{0}(r), \\
a_{2}^{\mathrm{BLM}} & \simeq-0.45 \beta_{0} z_{0}(r), \quad a_{3}^{\mathrm{BLM}} \simeq-0.21 \beta_{0}^{2} z_{0}(r), \quad a_{4}^{\mathrm{BLM}} \simeq+0.09 \beta_{0}^{3} z_{0}(r), \ldots
\end{array}
$$

This can be compared with the expansion in terms of pole masses:

$$
\begin{aligned}
\tilde{a}_{1} & \simeq-1.78 z_{0}(r), \quad \tilde{a}_{2}^{\text {non-BLM }} & \simeq 1.4 z_{0}(r), \\
\tilde{a}_{2}^{\mathrm{BLM}} & \simeq-1.92 \beta_{0} z_{0}(r), \quad \tilde{a}_{3}^{\mathrm{BLM}} & \simeq-2.8 \beta_{0}^{2} z_{0}(r), \quad \tilde{a}_{4}^{\mathrm{BLM}} \simeq-4.9 \beta_{0}^{3} z_{0}(r), \ldots
\end{aligned}
$$

(the second-order BLM coefficient in the pole mass scheme was first evaluated in Refs. [42] and its size interpreted as uncontrollable behavior of the perturbative series for the semileptonic widths). With this improvement, it fortunately does not make a too significant difference which approximation beyond the two-loop result to adopt, as illustrated below. Using the complete BLM-resummed result has the advantage of being nearly independent on the initial scale used to normalize $\alpha_{s}$. To this we add the second-order non-BLM correction evaluated with $\alpha_{s}=0.25$ and regard this as our central estimate: ${ }^{13}$

$$
A^{\text {pert }}(r ; \mu) \simeq 0.908 \text { at } \frac{\mu}{m_{b}}=\frac{1}{4.6} \text { and } r=0.0625
$$

Alternatively, if we keep only the full second-order result evaluated with $\alpha_{s}\left(m_{b}\right)=0.22$, this number becomes 0.909; including additionally third, or third and fourth BLM terms yields $A^{\text {pert }}(r ; \mu) \simeq 0.903$ and $A^{\text {pert }}(r ; \mu) \simeq 0.904$, respectively.

There are a few sources of possible theoretical uncertainties here. Before addressing them, we should emphasize that they shall not include uncertainties in the numerical values of $\alpha_{s}\left(m_{b}\right)$ or in the running quark masses - those are determined from experiment

\footnotetext{
${ }^{13}$ Since the infrared domain is removed to all orders in perturbation theory, the effective scale cannot be too low.
} 
and in principle have definite values as soon as the scheme is fixed, although they carry some error bars in practice.

The uncertainty in the second-order non-BLM coefficient even estimated conservatively [40] leads to $\delta A^{\text {pert }}$ about 0.0025 ; we may think it was actually overestimated. A potentially more significant uncertainty could come from varying the effective scale in $\alpha_{s}$ used to evaluate this term. However, since the magnitude of the non-BLM coefficient in Eq. (34) is about unity, this still would not exceed 0.003 .

The real question is then the size of the third- and even higher-order perturbative corrections. They are expected to be dominated by the BLM corrections which has been completely accounted for, and yield a sub-\% correction. Hence, we have reasons to consider the estimate of the theoretical uncertainty in knowledge of the perturbative factor $\delta A^{\text {pert }}(r ; 1 \mathrm{GeV}) \lesssim 0.009$ as conservative and justified:

$$
A^{\text {pert }}\left(0.25^{2} ; 1 \mathrm{GeV}\right)=0.908 \pm 0.009 \quad \text { at } \quad \alpha_{s}\left(m_{b}\right)=0.22
$$

The dependence on the actual value of $\alpha_{s}\left(m_{b}\right)$ follows from Eq. (6). The dependence on the values of heavy quark masses and the used normalization scale $\mu$ can be obtained by explicit evaluation of equations in Appendices A.2 and A.3.

\subsection{Wilson coefficients of power-suppressed operators}

While the Wilson coefficient of the kinetic operator is identical to the coefficient of the unit operator $A^{\text {pert }}$ and therefore known completely to two loops, the $\alpha_{s}$ corrections to the chromomagnetic operator have not been calculated so far. Once known, they would tell us the corrections to the coefficient for the $L S$ operator as well. The coefficient for the Darwin operator is an independent one, and its perturbative corrections are not known either. Part of the perturbative corrections comes from the short-distance renormalization of the heavy quark vector and axial current, and this one always enters as an overall factor. Therefore, it is reasonable to factor out $A^{\text {pert }}$ in the expression for the width in the absence of explicit calculations of the remaining corrections.

The accuracy of the tree level value of the exact chromomagnetic coefficient $1+A_{5}^{\text {pert }}$ is conservatively estimated as $30 \%$. It is generally expected to deteriorate for higher operators, and we assume only a $50 \%$ accuracy for the Darwin coefficient; since the latter is still poorly known and is rather constrained from above by data [6], this does not introduce a significant additional uncertainty. The chromomagnetic expectation value, on the contrary is known rather accurately, Eq. (17). The actual normalization scale for total semileptonic width is probably somewhat higher, which slightly decreases the expected effective value of $\mu_{G}^{2}$ in Eq. (1). We therefore estimate

$$
\frac{\delta_{A_{5}} \Gamma_{\mathrm{sl}}}{\Gamma_{\mathrm{sl}}} \simeq 0.01, \quad \frac{\delta_{D} \Gamma_{\mathrm{sl}}}{\Gamma_{\mathrm{sl}}} \lesssim 0.015
$$

Lack of evaluation of the $\mathcal{O}\left(\alpha_{s}\right)$ corrections to chromomagnetic and Darwin Wilson coefficients becomes now one of the limiting factors in the overall theoretical precision [26]. 


\section{$5 \quad$ Theoretical Uncertainties in $\Gamma\left(B \rightarrow X_{c} \ell \nu\right)$}

\subsection{First yet least: local duality violation}

A separate, more conceptual factor limiting the achievable theoretical accuracy would be violation of local duality. While it is related to high orders in the OPE, it has a different origin from what was discussed in Sect. 3.4 - a factorial growth of the expectation values of the higher-dimensional operators in the OPE, which makes the practical OPE series only asymptotic $[27,28]$. Both the qualitative pattern and numerical aspects of duality violation depend crucially on the observable in question.

In Ref. [43] a detailed and comprehensive analysis of limitations to local duality in $B \rightarrow X_{c} \ell \nu$ was given. Here we reiterate some of the salient points:

- Meaningful statements about duality violation require an accurate definition of the notion. That has been given for the OPE-treatable quantities like inclusive decay widths, and must be understood as deviations from the nonperturbatively corrected predictions through a sufficiently high order in the OPE (as opposed to parton-level estimates).

- Claims of duality violations one can find in the literature often turn out to constitute violations of the OPE. No example of the latter has been given so far, and actually are not expected to exist.

- On quite general grounds one infers that duality violations are given by 'oscillating' functions a lá $\sin \frac{m_{Q}}{\mu_{\text {hadr }}}$ that get further suppressed by powers of the energy release. No systematic excess or depletion of the rate compared to the OPE is allowed.

- The fully integrated width $\Gamma_{\mathrm{sl}}(B)$ relegates local duality violation per se to rather high orders. I.e., limitations of local duality are suppressed by a high power of the energy release. However, a much larger impact can exist on differential distributions or various widths with cuts.

- It has been estimated that limitations to duality in $\Gamma_{\mathrm{sl}}(B)$ amount to at most a few permill. This bound cannot be rigorously proved from QCD without some quantitative understanding of details of dynamics governing the nonperturbative regime of QCD. However, in any scenario effects of local duality violation in total semileptonic widths are much smaller than those in the differential distributions or moments used to extract the heavy quark parameters, thus introducing only negligible corrections in practice compared to the latter.

\subsection{Summary}

We have expressed the total semileptonic width of $B$ mesons as a functions of heavy quark parameters, namely quark masses and $B$ expectation values of local operators through order $1 / m_{Q}^{3}$, in addition to $\left|V_{c b}\right|^{2}$. The relation still has a potential theoretical error 
due to perturbative uncertainties in the Wilson coefficient of the leading $\bar{b} b$ operator, the QCD radiative corrections in the Wilson coefficients of chromomagnetic and Darwin operators, in the impact of 'intrinsic charm' and in the contributions from higher-order power suppressed contributions. As already stated in Eq.(7), we estimate their impact on $\left|V_{c b}\right|$ not to exceed $\pm 0.005, \pm 0.012, \pm 0.007$ and 0.004 , respectively. We think that these estimates are far from aggressive; the actual uncertainties might even be smaller. For instance, as mentioned in Sect. 6.1, there are correlations between $\alpha_{s}$ and the value extracted for $m_{Q}$. Furthermore, a large part of the contribution from the charm operators is accounted for by the Darwin contribution, when the latter is extracted from experiment, cf. Eq. (27). By the way, this "IC" contribution may explain why the central value of $\rho_{D}^{3}$ as suggested by experiment is smaller than anticipated.

In summary: we estimate that the present theoretical accuracy in expressing $\Gamma_{\mathrm{sl}}(B)$ through the heavy quark parameters corresponds to an uncertainty in $\left|V_{c b}\right|$ of about $2 \%$, Eq. (7).

\section{Determining the Heavy Quark Parameters}

Through order $1 / m_{Q}^{3}$ we have the following heavy quark parameters:

1. Quark masses $m_{b}(\mu)$ and $m_{c}(\mu)$;

2. The chromomagnetic and kinetic expectation values $\mu_{G}^{2}(\mu)$ and $\mu_{\pi}^{2}(\mu)$, respectively;

3. The Darwin and $L S$ terms $\rho_{D}^{3}(\mu)$ and $\rho_{L S}^{3}(\mu)$, respectively.

We have excluded the charm operators represented by $H_{c}$, which are treated separately. We want to determine the values of these HQ parameters in a way that does not jeopardize the strong points of the OPE expression for the width, Eq. (1), namely an expansion in inverse powers of (at worst) $m_{b}-m_{c}$, when only local operators are relevant.

The $b$ quark mass has been extracted from beauty production at threshold in $e^{+} e^{-}$ annihilation by several authors [44]. Their findings are completely consistent within the stated uncertainties of about 1.5\%. The techniques (and moments) employed in the analysis differ somewhat from author to author and the agreement in their findings is reassuring. One should keep in mind, though, that these determinations not only share their experimental input. The underlying approach is the same, as well as a number of general assumptions. The value stated in Eq. (15) could thus be subject to some systematic bias. Arguments based on the SV sum rules actually suggest that $m_{b}^{k i n}(1 \mathrm{GeV})$ lies a bit above $4.6 \mathrm{GeV}$, if a relatively low kinetic expectation value extracted from experiment so far is confirmed.

The values for $m_{b}$ and $m_{c}$ can be determined also from the shape of the energy spectra in semileptonic (or $b \rightarrow s+\gamma$ ) $B$ decays, which is most concisely encoded in their first few moments. Extractions were attempted based on the formulae of Ref. [45], their status is still controversial. Another relevant for us area of concern in the CLEO analysis is that the relation of Eq. (16) is imposed as a constraint for $m_{b}-m_{c}$ in semileptonic decays. 
Irrespective of that it should be noted that the central value extracted from $e^{+} e^{-} \rightarrow \bar{b} b$ and from $B$ decays agree well even within only the stated errors reported in the latter. Considering that the two processes have very different systematics experimentally as well as theoretically and should not have a correlation with the possible bias alluded to above, this is a nontrivial and encouraging result.

Since the early days of the HQE one has often employed the expansion given in Eq. (16) that relates the difference $m_{b}-m_{c}$ and the spin averaged charm and beauty meson masses. Yet in view of the accuracy in $m_{b}$ that has been achieved, the relation (16) may represents the most vulnerable part of the analysis. It suffers from two systematic weaknesses:

- It brings in effectively an expansion in $1 / m_{c}$ rather than $1 / m_{b}$ or $1 /\left(m_{b}-m_{c}\right)$. At the very least it is obvious that $1 /\left(m_{b}-m_{c}\right)^{2}$ and $1 / m_{c}^{2}$ differ a lot in magnitude, since a short-distance charm mass enters.

- It involves the nonlocal correlators $\rho_{\pi \pi}^{3}$ and $\rho_{s}^{3}$ intrinsically unrelated to $B$ decays. There are actually complementing indications that their sizes are particularly large. ${ }^{14}$

Accordingly one is ill-advised to impose relation (16) in the analysis. Instead one should check the validity of the relation if there is an option of determining $m_{c}$ independently. It is possible that Eq. (16) holds with reasonable accuracy; for eigenvalues of Hamiltonian often exhibit a more obedient behavior than formfactors etc. However we cannot count much on such good behavior a priori. Since $\mu_{\pi}^{2}-\mu_{G}^{2} \ll \mu_{\pi}^{2}$, the expansion

$$
\begin{gathered}
m_{b}-m_{c}=M_{B}-M_{D}+\frac{\mu_{\pi}^{2}-\mu_{G}^{2}}{2}\left(\frac{1}{m_{c}}-\frac{1}{m_{b}}\right)- \\
\frac{-\rho_{D}^{3}-\rho_{L S}^{3}+\bar{\rho}^{3}+\rho_{\pi G}^{3}+\rho_{A}^{3}}{4}\left(\frac{1}{m_{c}^{2}}-\frac{1}{m_{b}^{2}}\right)+\mathcal{O}\left(1 / m_{Q}^{3}\right),
\end{gathered}
$$

i.e., where the pseudoscalar rather than the spin averaged meson masses are used, is expected to be more stable with respect to higher order power corrections [14]. It requires some care incorporating the perturbative corrections to $\mu_{G}^{2}$, but is more promising in reducing the uncontrollable errors.

When the primary goal is to determine $\left|V_{c b}\right|$ as precisely as possible rather than the $\mathrm{HQ}$ parameters, another observation becomes important. Almost the same combination of the HQ parameters controls the low semileptonic moments and the total semileptonic width. It also controls the first hadronic moment $\left\langle M_{X}^{2}\right\rangle$. This combination of HQ parameters can thus be determined with higher accuracy than the individual HQ parameters - and the error associated with it becomes partially experimental. The theoretical status of evaluations of the moments is not as advanced as for $\Gamma_{\mathrm{sl}}$, therefore the expressions used for them should be viewed with lower confidence than what we know about the width itself. Nevertheless, we shall adopt them literally to illustrate the point.

\footnotetext{
${ }^{14}$ It was independently observed analyzing the actual hadron mass spectrum [14], in the exactly solvable 't Hooft model [46] and in the pilot lattice study [18].
} 


\subsection{Numerical examples}

An important new element is provided by the measurements of lepton energy and hadronic mass moments in inclusive semileptonic $B$ decays, as pioneered by CLEO and achieved also by DELPHI. Such data provide us with novel detailed information on the HQ parameters. Even though they do not allow to pinpoint the values of $m_{b}$ and $m_{c}$ separately, they turn out to improve significantly the accuracy and credibility of extracting $\left|V_{c b}\right|$. To illustrate this, we adopt evaluation Eqs. (5) and (6) neglecting possible uncertainty in $\alpha_{s}\left(m_{b}\right)$.

Comparing with the predictions for, say, the first lepton moment $\left\langle E_{\ell}\right\rangle$ or first hadronic moment $\left\langle M_{X}^{2}\right\rangle$ expressed in terms of the very same heavy quark parameters, we arrive at

$$
\begin{aligned}
\left|V_{c b}\right|=V_{c b}^{0}\left\{1-1.70 \mathrm{GeV}^{-1}\right. & \left(\left\langle E_{\ell}\right\rangle-1.383 \mathrm{GeV}\right)-0.075\left(m_{c}(1 \mathrm{GeV})-1.15 \mathrm{GeV}\right) \\
& +0.07\left(\mu_{\pi}^{2}-0.4 \mathrm{GeV}^{2}\right)-0.055\left(\rho_{D}^{3}-0.2 \mathrm{GeV}^{3}\right) \\
& \left.-0.085\left(\mu_{G}^{2}-0.35 \mathrm{GeV}^{2}\right)-0.005\left(\rho_{L S}^{3}+0.15 \mathrm{GeV}^{3}\right)\right\}, \\
\left|V_{c b}\right|=V_{c b}^{0}\left\{1+0.14 \mathrm{GeV}^{-2}\right. & \left(\left\langle M_{X}^{2}\right\rangle-4.54 \mathrm{GeV}^{2}\right)-0.03\left(m_{c}(1 \mathrm{GeV})-1.15 \mathrm{GeV}\right) \\
& +0.1\left(\mu_{\pi}^{2}-0.4 \mathrm{GeV}^{2}\right)+0.1\left(\rho_{D}^{3}-0.2 \mathrm{GeV}^{3}\right) \\
& \left.-0.01\left(\mu_{G}^{2}-0.35 \mathrm{GeV}^{2}\right)+0.006\left(\rho_{L S}^{3}+0.15 \mathrm{GeV}^{3}\right)\right\} .
\end{aligned}
$$

Taking for orientation the literal error bars on the two moments quoted by DELPHI [6] we would arrive at the uncertainties due to heavy quark parameters at the level of $2.5 \%$ and $1.2 \%$, respectively. To have an independent experimental constraints on kinetic and Darwin expectation values one can use the second and third hadronic moments, or their improved version [26].

The additional uncertainty would come from the charm expectation value $H_{c}$. However, as stated by Eq. (27), in such an approach it is expected to be milder: extracting in practice the Darwin expectation value, one partially accounts in it for the effects of possible contribution of $H_{c}$.

A similar reduction of sensitivity to $\alpha_{s}$ looks probable. According to Fig. 1 the effective scale of heavy quark masses shaping $\Gamma_{\mathrm{sl}}(b \rightarrow c)$ is somewhat larger than $1 \mathrm{GeV}$; if the same applies to the lowest moments, there should be a correlation between the extracted values of the masses and $\alpha_{s}$ reducing the dependence on the latter. This can be visualized by adopting a higher normalization scale for masses $\mu \simeq 1.5$ or even $2 \mathrm{GeV}$ which reduces the overall perturbative correction.

\section{Summary and Outlook}

The heavy quark expansion allows to express $\Gamma\left(B \rightarrow X_{c} \ell \nu\right)$ as a series in powers of $1 /\left(m_{b}-m_{c}\right)$ with coefficients that are controlled by the $B$ expectation values of local HQ operators; the number of unknowns here through order $1 / m_{b}^{3}$ is limited. Duality violations for this fully integrated rate have been estimated not to exceed the few permill level, i.e. to be irrelevant. Contributions from unknown higher order corrections - perturbative as 
well as nonperturbative - are also estimated to be near or below 1\%. It would require a very major effort to improve on this situation; at present we see no need for that, except computing the perturbative corrections to the chromomagnetic and Darwin operators.

The burning issue is how accurately we can determine the values of the HQ parameters from other observables. In the past the validity of the heavy quark expansion for $\Gamma(B \rightarrow$ $\left.X_{c} \ell \nu\right)$ has been compromised by invoking the relation of Eq. (16): $m_{b}-m_{c}$ is effectively given by an expansion in $1 / m_{c}$ with the additional drawback of uncontrolled non-local correlators contributing.

This weak link in the analysis can be overcome now: measuring the energy and mass moments in $B$ decays allows us to obtain values for the HQ parameters from expressions in powers of $1 /\left(m_{b}-m_{c}\right)$, where contributions from nonlocal operators are absent. Furthermore the HQ parameters the size of which previously could be inferred mainly by theoretical arguments, can be measured - and actually in more than one way. Such redundant extractions are a powerful tool in establishing theoretical control. It turns out that the low leptonic and first hadronic moments are controlled by almost the same combination of HQ parameters as $\Gamma\left(B \rightarrow X_{c} \ell \nu\right)$. This means that uncertainties previously viewed - correctly - as theoretical, become more experimental and thus can be reduced by better data. The situation there - while promising and encouraging - is still fluid requiring closer experimental as well as theoretical scrutiny. On the theoretical side higher order contributions to the moments have to be evaluated with the same care as it has been done in this paper for the total semileptonic $B$ width. On the experimental side it is essential that the cut in the lepton energy be kept as low as possible. For unless the major part of the spectrum is measured, limitations to local duality can enter through the 'back door' to haunt us. Furthermore such cuts decrease the hardness of the transition and thus deteriorate the convergence and reliability of the expansion.

Extracting $\left|V_{c b}\right|$ with neither the theoretical nor the experimental uncertainty exceeding the one percent level is thus within our reach. It appears unlikely that a higher accuracy should ever become necessary.

Finally - and this is one of the strengths of the OPE - the same values of the heavy quark parameters $m_{b}, \mu_{\pi}^{2}, \rho_{D}^{3}$ etc. can be used when extracting $\left|V_{u b}\right|$ from $B \rightarrow X_{u} \ell \nu$ transitions. The latter, however additionally require evaluation of the properly defined four-quark expectation values with $b$ and light quarks.

Acknowledgments: We have benefited from exchanges with S. Brodsky, S. Gardner, J. Kühn and A. Vainshtein. N.U. is also grateful to M. Battaglia, M. Calvi, P. Gambino and P. Roudeau for many related discussions. Three of us (D.B., I.B. and N.U.) are grateful for the hospitality extended to them by the Institut fur Theoretische Teilchenphysik, Universität Karlsruhe, where our collaboration was started. This work has been supported in part by the National Science Foundation under grant number PHY00-87419, by the DFG Research Group "Quantenfeldtheorie, Computeralgebra und Montre Carlo Simulationen", by the DFG Sonderforschungsbereich SFB/TR9 "Computational Particle Physics" and by the German Ministry for Education and Research BMBF under the grant number 05HT1VKB1 and the DFG Merkator Program. 


\section{Appendices}

\section{A.1 Heavy quark masses}

Having stated in Sect. 3.1 our preferences for which quark masses to adopt, we mention here how one can translate between the different 'quark mass languages'.

The usually used $\overline{\mathrm{MS}}$ mass $m_{Q}(\mu)$ is not really a quantity in the Lagrangian, it is rather a combination of parameters optimized for perturbative calculations in dimensional regularization. Its perturbative relation to the pole mass

$$
\begin{aligned}
m^{\text {pole }}= & \bar{m}(\bar{m})\left\{1+\frac{4}{3} \frac{\alpha_{s}^{\overline{\mathrm{MS}}}(\bar{m})}{\pi}+\left(\frac{\alpha_{s}^{\overline{\mathrm{MS}}}(\bar{m})}{\pi}\right)^{2}\left[\frac{\beta_{0}}{2}\left(\frac{\pi^{2}}{6}+\frac{71}{48}\right)\right.\right. \\
& \left.\left.+\frac{665}{144}+\frac{\pi^{2}}{18}\left(2 \ln 2-\frac{19}{2}\right)-\frac{1}{6} \zeta(3)-\frac{8}{3}\right]\right\}+\ldots
\end{aligned}
$$

is known now to three loops [47]. At $\mu \geq m_{Q}$ the $\overline{\mathrm{MS}}$ mass coincides more or less with the running Lagrangian mass probed at scale $\propto \mu$, and thus is appropriately used to describe high energy processes, $E \gtrsim m_{Q}$. However maintaining by definition the same $\mu$-dependence it becomes unphysical for $\mu \ll m_{Q}$,

$$
\bar{m}_{Q}(\mu) \simeq \bar{m}_{Q}\left(\bar{m}_{Q}\right)\left[1+\frac{2 \alpha_{s}}{\pi} \log \frac{m_{Q}}{\mu}\right]
$$

where actual running becomes much slower. The $\overline{\mathrm{MS}}$ mass is thus inappropriate for treating decay processes where the relevant scales are well below $m_{Q}$ and the evolution to a low normalization point $\mu \ll m_{Q}$ becomes crucial.

The kinetic mass in perturbation theory order by order is defined by subtracting from the pole mass the perturbative contributions of the heavy quark parameters:

$$
m_{b}(\mu)=m_{b}^{\text {pole }}-[\bar{\Lambda}(\mu)]_{\text {pert }}-\frac{\left[\mu_{\pi}^{2}(\mu)\right]_{\text {pert }}}{2 m_{b}(\mu)}
$$

where the latter are determined from the corresponding SV sum rules cut at energy $\epsilon=\mu$ :

$$
\bar{\Lambda}(\mu)_{\mathrm{pert}}=\frac{4}{3} C_{F} \frac{\alpha_{s}(M)}{\pi} \mu\left(1+\frac{\alpha_{s}}{\pi}\left[\frac{\beta_{0}}{2}\left(\ln \frac{M}{2 \mu}+\frac{8}{3}\right)-C_{A}\left(\frac{\pi^{2}}{6}-\frac{13}{12}\right)\right]+\ldots\right),
$$

with the standard notation $\bar{\Lambda} \equiv \lim _{m_{Q} \rightarrow \infty}\left[M_{H_{Q}}-m_{Q}\right]$, and

$$
\mu_{\pi}^{2}(\mu)_{\mathrm{pert}}=C_{F} \frac{\alpha_{s}(M)}{\pi} \mu^{2}\left(1+\frac{\alpha_{s}}{\pi}\left[\frac{\beta_{0}}{2}\left(\ln \frac{M}{2 \mu}+\frac{13}{6}\right)-C_{A}\left(\frac{\pi^{2}}{3}-\frac{13}{12}\right)\right]+\ldots\right),
$$

where $C_{F}=\frac{4}{3}$ and $C_{A}=N_{c}=3$, and $M$ denoting an arbitrary scale used to normalize $\alpha_{s}$. The latter is assumed in the $\overline{\mathrm{MS}}$ scheme in Eqs. (A.4) and (A.5). The thus defined mass exhibits only a linear in $\mu$ dependence on the scale,

$$
\frac{\mathrm{d} m_{Q}^{k i n}(\mu)}{\mathrm{d} \mu}=-\frac{16}{9} \frac{\alpha_{s}(\mu)}{\pi}-\frac{4}{3} \frac{\alpha_{s}(\mu)}{\pi} \frac{\mu}{m_{Q}}+\mathcal{O}\left(\alpha_{s}^{2}\right)
$$


compared to Eq. (A.2). The pole mass would corresponds to integrating this evolution down to $\mu=0$ at each particular order - alas it leads to an ill-defined result.

One could have extended the definition shown in Eq. (A.3) to include the corrections scaling like $\alpha_{s}^{k} \frac{\mu^{3}}{m_{Q}^{2}}$ and higher. Besides the known perturbative contribution in $\rho_{D}^{3}$ this requires the similar terms for the nonlocal expectation values $\rho_{\pi \pi}^{3}$ and $\rho_{S}^{3}$; the secondorder non-BLM pieces there have not been calculated. The one-loop effects, on the other hand, appear to cancel in the sum of all $\mu^{3} / m_{Q}^{2}$ terms, and the resulting shift beyond one loop (say, including evaluated BLM corrections) is tiny. In any case for the beauty mass this makes no visible difference whatsoever. There is an additional more subtle reason not to pay much attention to such terms. Therefore, we prefer to consider Eq. (A.3) as exact defining what is understood by the running masses, unless accuracy in their practical extraction will improve in the future by orders of magnitude.

Often "HQET parameters" like $\bar{\Lambda},-\lambda_{1}$ etc. are quoted rather than $\bar{\Lambda}(\mu), \mu_{\pi}^{2}(\mu)$ with appropriate $\mu$; they simply correspond to taking the limit $\mu \rightarrow 0$, and in this respect are the close relatives of the pole masses. Likewise they are not well-defined; moreover, the irreducible uncertainty and order-by-order instability in their values is of order unity compared to their magnitude. In concrete calculations it is often possible to identify their relation to well-defined Wilsonian expectation values. Yet one has to be aware that their values depend sensitively on the context in which they appear, and one cannot transfer them from one case to the next blindly. As a rule of thumb one has for routinely used first-order calculations with the second-order BLM improvement using $\alpha_{s}\left(m_{b}\right)$ coupling equated to 0.22

$$
\bar{\Lambda}_{\mathrm{HQET}} \simeq \bar{\Lambda}(1 \mathrm{GeV})-0.255 \mathrm{GeV}, \quad-\lambda_{1} \simeq \mu_{\pi}^{2}(1 \mathrm{GeV})-0.18(\mathrm{GeV})^{2} .
$$

Finally we quote the relation between the running $m_{Q}(\mu)$ and $\overline{\mathrm{MS}}$ mass $\bar{m}_{Q}\left(m_{Q}\right)$ including full $\alpha_{s}^{2}$ and third-order BLM corrections:

$$
\begin{aligned}
m_{b}(\mu)= & \bar{m}_{b}\left(\bar{m}_{b}\right)\left\{1+\frac{4}{3} \frac{\alpha_{s}^{\overline{\mathrm{MS}}}\left(m_{b}\right)}{\pi}\left(1-\frac{4}{3} \frac{\mu}{\bar{m}_{b}}-\frac{\mu^{2}}{2 \bar{m}_{b}^{2}}\right)\right. \\
+ & \left(\frac{\alpha_{s}^{\overline{\mathrm{MS}}}\left(m_{b}\right)}{\pi}\right)^{2}\left[\frac{\beta_{0}}{2}\left(\frac{\pi^{2}}{6}+\frac{71}{48}\right)+\frac{665}{144}+\frac{\pi^{2}}{18}\left(2 \ln 2-\frac{19}{2}\right)-\frac{1}{6} \zeta(3)-\frac{8}{3}\right. \\
& \left.\quad-\frac{\mu}{m_{b}}\left(\frac{8 \beta_{0}}{9}\left(\ln \frac{m_{b}}{2 \mu}+\frac{8}{3}\right)-\frac{8 \pi^{2}}{9}+\frac{52}{9}\right)-\frac{\mu^{2}}{m_{b}^{2}}\left(\frac{\beta_{0}}{3}\left(\ln \frac{m_{b}}{2 \mu}+\frac{13}{6}\right)-\frac{\pi^{2}}{3}+\frac{23}{18}\right)\right] \\
+ & \left.\quad-\frac{\beta_{0}}{2}\right)^{2} \frac{\mu^{2}}{m^{2}}\left[\left(\ln \frac{\alpha_{s}}{2 \mu}\right)^{3}\left\{\frac{2353}{2592}+\frac{13}{36} \pi^{2}+\frac{7}{6} \zeta(3)-\frac{16}{9} \frac{\mu}{m_{b}}\left[\left(\ln \frac{m_{b}}{2 \mu}+\frac{8}{3}\right)^{2}+\frac{67}{36}-\frac{\pi^{2}}{6}\right]\right\}\right.
\end{aligned}
$$

This can serve as a useful reference to translate between the kinetic and other possible low-scale running masses, along with the similar relation between the pole and kinetic masses. 


\section{A.2 Perturbative corrections in the Wilsonian approach}

Here we give relations allowing one to determine in practice perturbative coefficients in the approach employing the Wilsonian separation of large and small virtualities.

The starting point is the general short-distance expansion exemplified by Eq. (8) for $\Gamma_{\mathrm{sl}}(b \rightarrow c)$, or its practically employed form Eq. (1). As stressed in Sect. 2, it must be $\mu$-independent. Then one considers it in perturbation theory limited in practice to a particular order in $\alpha_{s}$ and $1 / m_{b}$. Requiring $\mu$-independence completely defines the $\mu$-dependence of the Wilson coefficients once the $\mu$-dependence of masses and matrix elements in perturbation theory is known $[9,12]$. For truncated perturbation theory the actual expression has a residual dependence on $\mu$ to the uncalculated orders in $\alpha_{s}$. On the other hand, in the finite-order perturbation theory one can formally put $\mu=0$. This would set all the power-suppressed expectation values to zero and yield pole masses, hence fixing the perturbative series for $A^{\text {pert }}(r ; 0)$ to be the 'conventional' perturbative corrections in the pole mass scheme. This provides the initial condition for perturbative $\mu$-dependence at $\mu=0$.

To give the practical implementation for $\Gamma_{\mathrm{sl}}(b \rightarrow c)$ we need to specify more precisely our definition in Eq. (33): now we write

$$
z_{0}(r) A^{\text {pert }}(r ; \mu)=z_{0}(r)+a_{1}(r ; \mu) \frac{\alpha_{s}}{\pi}+a_{2}(r ; \mu)\left(\frac{\alpha_{s}}{\pi}\right)^{2}+a_{3}(r ; \mu)\left(\frac{\alpha_{s}}{\pi}\right)^{3}+\ldots
$$

and use the short-hand notations $a_{k}^{(0)}(r) \equiv a_{k}(r, 0)$ for the perturbative coefficients at $\mu=0$ (pole mass scheme). Then one has

$$
\begin{aligned}
a_{1}(r ; \mu)= & a_{1}^{(0)}(r)+\left[5 \Lambda_{1}+3 p_{1}\right] z_{0}(r)-\rho_{1} D(r)+\left[2(\sqrt{r}-r) \Lambda_{1}+(1-r) p_{1}\right] \frac{\mathrm{d} z_{0}(r)}{\mathrm{d} r}(\mathrm{~A} .10) \\
a_{2}(r ; \mu)= & a_{2}^{(0)}(r)+\left[5 \Lambda_{1}+3 p_{1}-\rho_{1} \frac{D(r)}{z_{0}(r)}\right] a_{1}^{(0)}(r)+\left[2(\sqrt{r}-r) \Lambda_{1}+(1-r) p_{1}\right] \frac{\mathrm{d} a_{1}^{(0)}(r)}{\mathrm{d} r} \\
+ & {\left[5 \Lambda_{2}+3 p_{2}+10 \Lambda_{1}^{2}+4 p_{1}^{2}+\frac{25}{2} \Lambda_{1} p_{1}-\left(\rho_{2}+\rho_{1}\left(5 \Lambda_{1}+\frac{7}{2} p_{1}\right)\right) \frac{D(r)}{z_{0}(r)}\right] z_{0}(r)+\frac{\rho_{1}^{2} D^{2}(r)}{z_{0}(r)} } \\
+ & {\left[2(\sqrt{r}-r) \Lambda_{2}+(1-r) p_{2}+(1+6 \sqrt{r}-7 r) \Lambda_{1}^{2}+\left(\frac{1}{4 r}+2-\frac{9}{4} r\right) p_{1}^{2}+\right.} \\
& \left.\left(\frac{1}{\sqrt{r}}+3+4 \sqrt{r}-8 r\right) \Lambda_{1} p_{1}\right] \frac{\mathrm{d} z_{0}(r)}{\mathrm{d} r}+\frac{1}{2}\left[2(\sqrt{r}-r) \Lambda_{1}+(1-r) p_{1}\right]^{2} \frac{\mathrm{d}^{2} z_{0}(r)}{\mathrm{d} r^{2}} \\
& -\rho_{1}\left[2(\sqrt{r}-r) \Lambda_{1}+(1-r) p_{1}\right] \frac{\mathrm{d} D(r)}{\mathrm{d} r}
\end{aligned}
$$

and the coefficient $a_{1}^{(0)}(r)$ is given by $[48,49]$

$$
\begin{aligned}
a_{1}^{(0)}(r) & =\frac{1}{24}\left[75-12 \pi^{2}-956 r-192 \pi^{2} r^{2}+956 r^{3}-3\left(25+4 \pi^{2}\right) r^{4}\right. \\
& +384 \pi^{2} r^{\frac{3}{2}}(1+r)+4\left(-17+64 r-64 r^{3}+17 r^{4}\right) \ln (1-r) \\
& -4 r\left(60+270 r-4 r^{2}+17 r^{3}-384 \sqrt{r}(1+r) \ln (1+\sqrt{r})\right) \ln (r)
\end{aligned}
$$




$$
\begin{aligned}
& +48\left(1-16 r^{\frac{3}{2}}+30 r^{2}-16 r^{\frac{5}{2}}+r^{4}\right) \ln (1-r) \ln r-12 r^{2}\left(36+r^{2}\right) \ln ^{2} r \\
& \left.-3072 r^{\frac{3}{2}}(1+r) \operatorname{Li}_{2}(\sqrt{r})+18\left(4+64 r^{2}+4 r^{4}+\frac{128 r^{\frac{3}{2}}(1+r)}{3}\right) \operatorname{Li}_{2}(r)\right] .
\end{aligned}
$$

The combinations $\Lambda_{1,2}, p_{1,2}$ and $\rho_{1,2}$ are the coefficients of the perturbative expansion of $\frac{1}{m_{b}}[\Lambda(\mu)]_{\text {pert }}$ and $\frac{1}{m_{b}^{2}}\left[\mu_{\pi}^{2}(\mu)\right]_{\text {pert }}$ in Eqs. (A.4) and (A.5) and of $\frac{1}{m_{b}^{3}}\left[\rho_{D}^{3}(\mu)\right]_{\text {pert }}$, to first and second orders in $\alpha_{s}$, respectively:

$$
\begin{array}{rlrl}
\Lambda_{1} & =\frac{4}{3} C_{F} \frac{\mu}{m_{b}}, & \Lambda_{2} & =\Lambda_{1}\left[\frac{\beta_{0}}{2}\left(\ln \frac{M}{2 \mu}+\frac{8}{3}\right)-C_{A}\left(\frac{\pi^{2}}{6}-\frac{13}{12}\right)\right], \\
p_{1}=C_{F} \frac{\mu^{2}}{m_{b}^{2}}, & p_{2}=p_{1}\left[\frac{\beta_{0}}{2}\left(\ln \frac{M}{2 \mu}+\frac{13}{6}\right)-C_{A}\left(\frac{\pi^{2}}{6}-\frac{13}{12}\right)\right], \\
\rho_{1}=\frac{2}{3} C_{F} \frac{\mu^{3}}{m_{b}^{3}}, & \rho_{2}=\rho_{1}\left[\frac{\beta_{0}}{2}\left(\ln \frac{M}{2 \mu}+2\right)-C_{A}\left(\frac{\pi^{2}}{6}-\frac{13}{12}\right)\right] .
\end{array}
$$

It should be noted that we did not include here shifts due to the spin-singlet $1 / m_{b}$ pieces of the kinetic and chromomagnetic operators (we have computed them to order $\alpha_{s}$ and to all orders in BLM). These corrections are $1 / m_{b}^{3}$ and governed by the full $1 / 2 m_{b}$ scale, hence totally insignificant in practice. We did include the effect of the Darwin operator to two full loops (modulo uncalculated $\mathcal{O}\left(\alpha_{s}\right)$ piece $A_{D}^{\text {pert }}$ ), since its coefficient is enhanced and strongly dominates over the $1 / m_{b}^{3}$ effects.

The improvement by the Wilsonian cutoff of the resulting perturbative series can be illustrated by comparing them at $\mu=1 \mathrm{GeV}$

$$
A_{\text {pert }}(1 \mathrm{GeV}) \simeq 1-0.94 \frac{\alpha_{s}\left(m_{b}\right)}{\pi}-5.1\left(\frac{\alpha_{s}\left(m_{b}\right)}{\pi}\right)^{2}-17\left(\frac{\alpha_{s}\left(m_{b}\right)}{\pi}\right)^{3}+63\left(\frac{\alpha_{s}\left(m_{b}\right)}{\pi}\right)^{4}+\ldots
$$

with those in the 'pole' (HQET) scheme:

$$
A_{\mathrm{pert}}^{\mathrm{pole}} \simeq 1-1.78 \frac{\alpha_{s}\left(m_{b}\right)}{\pi}-15.8\left(\frac{\alpha_{s}\left(m_{b}\right)}{\pi}\right)^{2}-230\left(\frac{\alpha_{s}\left(m_{b}\right)}{\pi}\right)^{3}-3640\left(\frac{\alpha_{s}\left(m_{b}\right)}{\pi}\right)^{4}-\ldots
$$

where numbers refer to $m_{c} / m_{b}=0.25$.

\section{A.3 BLM summation with Wilsonian cutoff}

The BLM correction in practice are simpler to calculate directly, using the generalized order- $\alpha_{s}$ relation (A.10) between Wilson coefficients with and without the cutoff. The technique of BLM resummation was recently reviewed in this context in Ref. [5]. It requires computing one-loop corrections with a non-zero gluon mass $\lambda$. The expressions for the one-loop correction to the perturbative width with non-zero gluon mass are rather lengthy and can be found in Ref. [50]. One also needs similar terms in $[\bar{\Lambda}(\mu)]^{\text {pert }},\left[\mu_{\pi}^{2}\right]^{\text {pert }}$ 
and $\left[\rho_{D}^{3}\right]^{\text {pert }}$. These are calculated by integrating the SV spectral density of Ref. [12] with the proper power of energy and are given as follows:

$$
\begin{aligned}
& \Lambda_{1}(\mu ; \lambda)=\frac{16 \alpha_{s}}{9 \pi} \frac{1}{m_{b}} \vartheta\left(\mu^{2}-\lambda^{2}\right)\left[\left(1-\frac{\lambda^{2}}{4 \mu^{2}}\right) \sqrt{\mu^{2}-\lambda^{2}}-\frac{3 \pi}{8} \lambda+\frac{3}{4} \lambda \arcsin \frac{\lambda}{\mu}\right], \\
& p_{1}(\mu ; \lambda)=\frac{4 \alpha_{s}}{3 \pi} \frac{1}{m_{b}^{2}} \vartheta\left(\mu^{2}-\lambda^{2}\right) \frac{\left(\mu^{2}-\lambda^{2}\right)^{3 / 2}}{\mu}, \\
& \rho_{1}(\mu ; \lambda)=\frac{8 \alpha_{s}}{9 \pi} \frac{1}{m_{b}^{3}} \vartheta\left(\mu^{2}-\lambda^{2}\right)\left[\sqrt{\mu^{2}-\lambda^{2}}\left(1+\frac{\lambda^{2}}{2 \mu^{2}}\right)-\frac{3 \lambda^{3}}{2}\left(\frac{\pi}{2}-\arcsin \frac{\lambda}{\mu}\right)\right] .
\end{aligned}
$$

One then has

$$
\begin{aligned}
a_{1}(r ; \mu ; \lambda)=a_{1}^{(0)}(r ; \lambda) & +\left[5 \Lambda_{1}(r ; \mu ; \lambda)+3 p_{1}(r ; \mu ; \lambda)\right] z_{0}(r)-\rho_{1}(r ; \mu ; \lambda) D(r) \\
+ & {\left[2(\sqrt{r}-r) \Lambda_{1}(r ; \mu ; \lambda)+(1-r) p_{1}(r ; \mu ; \lambda)\right] \frac{\mathrm{d} z_{0}(r)}{\mathrm{d} r} }
\end{aligned}
$$

The perturbative coefficients are explicitly given by the series

$$
\begin{aligned}
& A^{\mathrm{BLM}}(r ; \mu)=1+a_{1}(r ; \mu ; 0) \frac{\alpha_{s}\left(m_{b}\right)}{\pi}+\sum_{n=0}^{\infty} \frac{4}{\beta_{0}}\left(\frac{\beta_{0} \alpha_{s}\left(m_{b}\right)}{4 \pi}\right)^{n+2} \times \\
& \sum_{k=0}^{\frac{n}{2}}\left(-\pi^{2}\right)^{k} C_{n+1}^{2 k+1} \cdot \int \frac{\mathrm{d} \lambda^{2}}{\lambda^{2}}\left[\ln \frac{m_{b}^{2}}{\lambda^{2}}+\frac{5}{3}\right]^{n-2 k}\left(a_{1}(r ; \mu ; 0) \frac{m_{b}^{2}}{m_{b}^{2}+e^{-5 / 3} \lambda^{2}}-a_{1}\left(r ; \mu ; \lambda^{2}\right)\right) \mathrm{A}
\end{aligned}
$$

and the resummed result reads as

$$
\begin{aligned}
A^{\mathrm{BLM}}(r ; \mu)=1 & +a_{1}(r ; \mu ; 0) \frac{\alpha_{s}\left(m_{b}\right)}{\pi} \\
+\int_{-\infty}^{\infty} \mathrm{d} t & \frac{\frac{\beta_{0}}{4}\left(\frac{\alpha_{s}}{\pi}\right)^{2}}{\left(1+\frac{\beta_{0} \alpha_{s}}{4 \pi}\left(t-\frac{5}{3}\right)\right)^{2}+\left(\frac{\beta_{0}}{4} \alpha_{s}\right)^{2}}\left(a_{1}(r ; \mu ; 0) \frac{1}{1+e^{t-\frac{5}{3}}}-a_{1}\left(r ; \mu ; e^{t} m_{b}^{2}\right)\right) \\
& -\frac{4}{\beta_{0}}\left[\frac{m_{b}^{2}}{m_{b}^{2}-\Lambda_{V}^{2}} a_{1}(r ; \mu ; 0)-a_{1}\left(r ; \mu ;-\Lambda_{V}^{2}\right)\right]
\end{aligned}
$$

with

$$
\Lambda_{V}^{2}=m_{b}^{2} e^{-\frac{4 \pi}{\beta_{0} \alpha_{s}\left(m_{b}\right)}+\frac{5}{3}} .
$$

Numerically at $m_{c} / m_{b}=0.25, \mu / m_{b}=1 / 4.6$ and $\alpha_{s}\left(m_{b}\right)=0.22$ we obtain

$$
A^{\mathrm{BLM}}(r ; \mu)=0.915 \text {. }
$$




\section{References}

[1] I. Bigi, M. Shifman and N.G. Uraltsev, Ann. Rev. Nucl. Part. Sci. 47 (1997) 591.

[2] I. Bigi, M. Shifman, N. Uraltsev and A. Vainshtein, Phys. Rev. Lett. 71 (1993) 496.

[3] M. Gremm and A. Kapustin, Phys. Rev. D55 (1997) 6924.

[4] A. Sirlin, Nucl. Phys. B71 (1974) 29, and Rev. Mod. Phys. 50 (1978) 573.

[5] N. Uraltsev, Int. Journ. Mod. Phys. Lett. A17 (2002) 2317.

[6] M. Battaglia et al., hep-ph/0210319.

[7] N. Uraltsev, in Boris Ioffe Festschrift "At the Frontier of Particle Physics -- Handbook of QCD", Ed. M. Shifman (World Scientific, Singapore, 2001), Vol.3, p. 1577; hep-ph/0010328.

[8] I. Bigi, M. Shifman, N. Uraltsev and A. Vainshtein, Phys. Rev. D56 (1997) 4017.

[9] I.I. Bigi, M. Shifman, N.G. Uraltsev and A. Vainshtein, Phys. Rev. D52 (1995) 196.

[10] I. Bigi, N. Uraltsev and A. Vainshtein, Phys. Lett. B293 (1992) 430.

[11] I. Bigi, S. Brodsky, S. Gardner and N. Uraltsev, paper in preparation.

[12] N.G. Uraltsev, Nucl. Phys. B491 (1997) 303.

[13] A. Hoang, hep-ph/0204299.

[14] N. Uraltsev, Phys. Lett. B545 (2002) 337.

[15] I. Bigi, M. Shifman, N. Uraltsev and A. Vainshtein, Phys. Rev. D50 (1994) 2234.

[16] B. Blok and M. Shifman, Nucl. Phys. B399 (1993) 441 and 459.

[17] B. Blok and M. Lublinsky, Phys. Rev. D57 (1998) 2676.

[18] A. S. Kronfeld and J. N. Simone, Phys. Lett. B490, 228 (2000) 228 [Erratum-ibid. B495, (2000) 441].

[19] I.I. Bigi and N.G. Uraltsev, Nucl. Phys. B 423 (1994) 33.

[20] I. Bigi and N. Uraltsev, Phys. Lett. B280 (1992) 271.

[21] M. Franz, M.V. Polyakov and K. Goeke, Phys. Rev. D62 (2000) 074024.

[22] N. Uraltsev, Int. Journ. Mod. Phys. A14 (1999) 4641. 
[23] S. J. Brodsky and S. Gardner, Phys. Rev. D65 054016 (2002);

S. J. Brodsky, P. Hoyer, C. Peterson and N. Sakai, Phys. Lett. B93 451 (1980);

S. J. Brodsky, C. Peterson and N. Sakai, Phys. Rev. D23 2745 (1981).

[24] R.D. Dikeman and N.G. Uraltsev, Nucl. Phys. B509 (1998) 378;

I. Bigi, R.D. Dikeman and N. Uraltsev, Eur. Phys. J. C4 (1998) 453.

[25] R. A. Briere et al. [CLEO Collaboration], hep-ex/0209024.

[26] N. Uraltsev, hep-ph/0210044, Proc. ICHEP-2002, Amsterdam, the Netherlands.

[27] M. Shifman, Lectures on Heavy Quarks in Quantum Chromodynamics, in QCD and Beyond, Proc. Theoretical Advanced Study Institute in Elementary Particle Physics (TASI 95), Ed. D.E. Soper (World Scientific, Singapore, 1996), p. 409; hep$\mathrm{ph} / 9510377$.

[28] B. Chibisov, R. Dikeman, M. Shifman and N.G. Uraltsev, Int. Journ. Mod. Phys. A12 (1997) 2075.

[29] A. Czarnecki, K. Melnikov and N. Uraltsev, Phys. Rev. Lett. 80 (1998) 3189 and hep-ph/9708372.

[30] B. Aubert et al. [BABAR Collaboration], hep-ex/0207084.

[31] M. Calvi [DELPHI Collaboration], hep-ex/0210046.

[32] A. Le Yaouanc et al., Phys. Lett. B517 (2001) 135.

[33] C. Jin, hep-ph/9906212.

[34] I. Bigi, M. Shifman, N. Uraltsev, A. Vainshtein, Int. J. Mod. Phys. A9 (1994) 2467.

[35] S.J. Brodsky, G.P. Lepage and P.B. Mackenzie, Phys. Rev. D28 (1983) 228; G.P. Lepage and P.B. Mackenzie, Phys. Rev. D48 (1993) 2250.

[36] P. Ball, M. Beneke and V.M. Braun, Nucl. Phys. B452 (1995) 563.

[37] Yu.L. Dokshitzer, G. Marchesini and B.R. Webber, Nucl. Phys. B469 (1996) 93.

[38] S.J. Brodsky, E. Gardi, G. Grunberg and J. Rathsman, Phys. Rev. D63 (2001) 094017.

[39] T. van Ritbergen, Phys. Lett. B454 (1999) 353.

[40] A. Czarnecki and K. Melnikov, Phys. Rev. Lett. 78 (1997) 3630; Phys. Rev. D59 (1999) 014036.

[41] N.G. Uraltsev, Int. J. Mod. Phys. A11 (1996) 515.

[42] M. Luke, M. Savage and M. Wise Phys. Lett. B343 (1995) 329 and B345 (1995) 301. 
[43] I. Bigi and N. Uraltsev, Int. J. Mod. Phys. A16 (2001) 5201.

[44] K. Melnikov and A. Yelkhovsky, Phys. Rev. D59 (1999) 114009;

A. Hoang, Phys. Rev. D61 (2000) 034005;

M. Beneke and A. Signer, Phys. Lett. B471 (1999) 233;

J.H. Kuhn and M. Steinhauser, Nucl. Phys. B619 588 (2001) [Erratum-ibid. B640 415 (2002)] [arXiv:hep-ph/0109084].

[45] M. Gremm, A. Kapustin, Z. Ligeti and M. Wise, Phys. Rev. Lett. 77 (1996) 20;

A. Falk, M. Luke and M. Savage, Phys. Rev. D53 (1996) 2491 and D53 (1996) 6316;

A. Kapustin and Z. Ligeti, Phys. Lett. B355 (1995) 318;

A. Falk and M. Luke Phys.Rev. Phys. Rev. D57 (1998) 424;

Z. Ligeti, M. Luke, A. Manohar and M. Wise, Phys. Rev. D60 (1999) 034019.

[46] R. Lebed and N. Uraltsev, Phys. Rev. D62 (2000) 094011.

[47] K. Melnikov and T. van Ritbergen, Phys. Lett. B482 (2000) 99.

[48] S. Berman, Phys. Rev. 112 (1958) 267;

T. Kinoshita and A. Sirlin, Phys. Rev. 113 (1959) 1652.

[49] Y. Nir, Phys. Lett. B221 (1989) 184.

[50] P. Ball, M. Beneke and V. Braun, Phys. Rev. D52 (1995) 3929. 